\begin{tabular}{|c|l|}
\hline Title & $\begin{array}{l}\text { Temperature dependence of spin-dependent tunneling conductance of magnetic tunnel junctions with half-metall lic } \\
\text { Co2MnSi electrodes }\end{array}$ \\
\hline Author(s) & $\begin{array}{l}\text { Hu, Bing; Moges, Kidist; Honda, Y usuke; Liu, Hong-xi; Uemura, Tetsuy a; Y amamoto, Masafumi; Inoue, Jun-ichiro; } \\
\text { Shirai, Masafumi }\end{array}$ \\
\hline Citation & $\begin{array}{l}\text { Physical Review B, 94(9), 094428 } \\
\text { https://doi.org/10.1103/PhysRevB.94.094428 }\end{array}$ \\
\hline Issue Date & 2016-09-23 \\
\hline Doc URL & http://hdl.handle.net/2115/63561 \\
\hline Rights & O2016 A merican Physical Society \\
\hline Type & article \\
\hline File Information & PhysRevB.94.094428.pdf \\
\hline
\end{tabular}

Instructions for use 


\title{
Temperature dependence of spin-dependent tunneling conductance of magnetic tunnel junctions with half-metallic $\mathrm{Co}_{2} \mathrm{MnSi}$ electrodes
}

\author{
Bing Hu, Kidist Moges, Yusuke Honda, Hong-xi Liu, Tetsuya Uemura, and Masafumi Yamamoto* \\ Division of Electronics for Informatics, Graduate School of Information Science and Technology, \\ Hokkaido University, Sapporo 060-0814, Japan \\ Jun-ichiro Inoue \\ Department of Applied Physics, Nagoya University, Nagoya 464-8603, Japan
}

Masafumi Shirai

Research Institute of Electrical Communication, Tohoku University, Sendai 980-8577, Japan

and Center for Spintronics Research Network, Tohoku University, Sendai 980-8577, Japan

(Received 14 June 2016; revised manuscript received 16 August 2016; published 23 September 2016)

\begin{abstract}
In order to elucidate the origin of the temperature $(T)$ dependence of spin-dependent tunneling conductance $(G)$ of magnetic tunnel junctions (MTJs), we experimentally investigated the $T$ dependence of $G$ for the parallel and antiparallel magnetization alignments, $G_{\mathrm{P}}$ and $G_{\mathrm{AP}}$, of high-quality $\mathrm{Co}_{2} \mathrm{MnSi}(\mathrm{CMS}) / \mathrm{MgO} / \mathrm{CMS}$ MTJs having systematically varied spin polarizations $(P)$ at $4.2 \mathrm{~K}$ by varying the $\mathrm{Mn}$ composition $\alpha$ in $\mathrm{Co}_{2} \mathrm{Mn}_{\alpha} \mathrm{Si}$ electrodes that exhibited giant tunneling magnetoresistance ratios. Results showed that $G_{\mathrm{P}}$ normalized by its value at $4.2 \mathrm{~K}$ exhibited a notable, nonmonotonic $T$ dependence although its variation with $T$ was significantly smaller than that of $G_{\mathrm{AP}}$ normalized by its value at $4.2 \mathrm{~K}$, indicating that an analysis of the experimental $G_{\mathrm{P}}(T)$ is critical to revealing the origin of the $T$ dependence of $G$. By analyzing the experimental $G_{\mathrm{P}}(T)$, we clarified that both spin-flip inelastic tunneling via a thermally excited magnon and spin-conserving elastic tunneling in which $P$ decays with increasing $T$ play key roles. The experimental $G_{\mathrm{AP}}(T)$, including its stronger $T$ dependence for higher $P$ at $4.2 \mathrm{~K}$, was also consistently explained with this model. Our findings provide a unified picture for understanding the origin of the $T$ dependence of $G$ of MTJs with a wide range of $P$, including MTJs with high $P$ close to a half-metallic value.
\end{abstract}

DOI: 10.1103/PhysRevB.94.094428

\section{INTRODUCTION}

Spintronic devices, which utilize the spin degree of freedom in addition to the charge of the electron, have attracted much interest as future-generation electron devices. This is because of their potential advantages of nonvolatility, decreased power consumption, and reconfigurable logic function capabilities $[1,2]$. A highly efficient spin source is essential for constructing spintronic devices. Half-metallic ferromagnets (HMFs) are one of the most suitable spin source materials because of their complete spin polarization at the Fermi level $\left(E_{\mathrm{F}}\right)$ arising from an energy gap for one spin direction (mostly the minority-spin band) [3]. Co-based Heusler alloys $\left(\mathrm{Co}_{2} Y Z\right.$, where $Y$ is usually a transition metal and $Z$ is a main group element) [4] are among the most extensively applied to spintronic devices, including magnetic tunnel junctions (MTJs) [5-17] and giant magnetoresistance (GMR) devices [18-22], and for spin injection into semiconductors [23-27]. This is because of the HMF nature theoretically predicted for many of these alloys [28-31] and because of their high Curie temperatures, which are well above room temperature [32].

$\mathrm{Co}_{2} \mathrm{MnSi}(\mathrm{CMS})$ is one of the most extensively investigated ferromagnetic electrode materials among the $\mathrm{Co}_{2} \mathrm{YZ}$ family [6-12,18,20,23,26,27,33-41]. This is because of its theoretically predicted half-metallic nature with a large half-metal

*yamamoto@nano.ist.hokudai.ac.jp gap of $0.81 \mathrm{eV}$ for its minority-spin band [29-31] and its high Curie temperature of $985 \mathrm{~K}$ [32]. In practice, to various degrees, off-stoichiometry in $\mathrm{Co}_{2} \mathrm{YZ}$ thin films prepared by magnetron sputtering or molecule beam epitaxy is inevitable, leading to structural defects. Picozzi et al. predicted from first principles that half-metallicity in $\mathrm{CMS}$ and $\mathrm{Co}_{2} \mathrm{MnGe}$ (CMG) is lost for $\mathrm{Co}_{\mathrm{Mn}}$ antisites, where a $\mathrm{Mn}$ site is replaced by a Co atom, because of the appearance of minority-spin in gap states near $E_{\mathrm{F}}$, while half-metallicity is retained for $\mathrm{Mn}_{\mathrm{Co}}$ antisites, where a Co site is replaced by a $\mathrm{Mn}$ atom [33]. The effect of off-stoichiometry on the half-metallicity of CMS and CMG has been systematically investigated recently using various experimental approaches, including the tunneling magnetoresistance (TMR) ratios of MTJs [9-12], the saturation magnetization per formula unit of thin films [12], the surface spin polarization [42], the magnetic states as investigated by $\mathrm{x}$-ray absorption spectroscopy and $\mathrm{x}$-ray magnetic circular dichroism [43-45], and the electronic states as investigated through spin-resolved low-energy and hard $\mathrm{X}$-ray photoelectron spectroscopy [46-48]. These experimental studies, along with first-principles calculations, demonstrated that $\mathrm{Co}_{\mathrm{Mn}}$ antisites induced by a Mn-deficient composition (the Mn composition $\alpha<2-\beta$ in the composition expression of $\mathrm{Co}_{2} \mathrm{Mn}_{\alpha} \mathrm{Si}_{\beta}$ ) is detrimental to the half-metallicity of CMS. Furthermore, it was shown that harmful $\mathrm{Co}_{\mathrm{Mn}}$ antisites can be suppressed and half-metallicity enhanced by preparing CMS thin films with a Mn-rich composition [9-12]. It was also shown that $(\mathrm{Mn}+\mathrm{Fe})$-rich compositions are critical 
to suppressing these harmful antisites and to retaining the half-metallic electronic states for $\mathrm{Co}_{2}(\mathrm{Mn}, \mathrm{Fe}) \mathrm{Si}$ (CMFS) quaternary alloys $[16,17]$. Given these findings, we demonstrated giant TMR ratios of up to $1995 \%$ at $4.2 \mathrm{~K}$ and up to $354 \%$ at $290 \mathrm{~K}$ for $\mathrm{CMS} / \mathrm{MgO} / \mathrm{CMS}$ MTJs (CMS MTJs) having Mn-rich CMS electrodes [11] and up to $2610 \%$ at $4.2 \mathrm{~K}$ and $429 \%$ at $290 \mathrm{~K}$ for CMFS/MgO/CMFS MTJs (CMFS MTJs) with Mn-rich, lightly Fe-doped CMFS electrodes [16].

To take full advantage of the half-metallic nature of Co-based Heusler alloys as spin source materials at room temperature, it is important to clarify the origin of the temperature $(T)$ dependence of spin-dependent tunneling conductance $(G)$ of MTJs. The $T$ dependence of $G$ for the parallel and antiparallel magnetization alignments, $G_{\mathrm{P}}$ and $G_{\mathrm{AP}}$, of MTJs has been discussed on the basis of models proposed by Zhang et al. [49] and by Shang et al. [50]. Zhang et al. accounted for the $T$ dependence of $G_{\mathrm{P}}$ and $G_{\mathrm{AP}}$ by spin-flip inelastic tunneling via a thermally excited magnon while assuming a $T$-independent spin polarization, $P$ [49]. The Zhang model predicts that (i) both $G_{\mathrm{P}}$ and $G_{\mathrm{AP}}$ increase with increasing $T$, and (ii) the increase in $G_{\mathrm{AP}}$ normalized by its value at $4.2 \mathrm{~K}\left[G_{\mathrm{AP}}(T) / G_{\mathrm{AP}}(4.2 \mathrm{~K})\right]$ with increasing $T$ becomes larger for higher $P$ while that in $G_{\mathrm{P}}$ normalized by its value at $4.2 \mathrm{~K}\left[G_{\mathrm{P}}(T) / G_{\mathrm{P}}(4.2 \mathrm{~K})\right]$ with $T$ becomes smaller for higher $P$. On the other hand, Shang et al. took into consideration only spin-conserving elastic tunneling with a decaying $P$ with increasing $T$. The Shang model predicts that $G_{\mathrm{AP}}$ increases with $T$, of which the effect on $G_{\mathrm{AP}}(T)$ is apparently in agreement with that of the Zhang model. Indeed, the experimental $G_{\mathrm{AP}}(T)$ had been explained and fitted by the original Zhang model in [51,52] or by the Shang model in [53]. However, note that the tunneling mechanisms that determine the $T$ dependence are intrinsically different between these two models. On the other hand, the Shang model predicts that $G_{\mathrm{P}}$ decreases, which is opposed to the prediction of the Zhang model. Previous studies on MTJs with an amorphous $\mathrm{AlO}_{x}$ tunnel barrier reported an increase in $G_{\mathrm{P}}$ with increasing $T$ up to room temperature [50,51]. Most of the epitaxial MTJs with an $\mathrm{MgO}$ or $\mathrm{MgAl}_{2} \mathrm{O}_{4}$ tunnel barrier that have been reported so far have also shown an increase in $G_{\mathrm{P}}[8,52-57]$, while some epitaxial MTJs showed a decrease [58-60]. The increase in $G_{\mathrm{P}}$ with $T$ for MTJs, including epitaxial MTJs, has been mostly explained by spin-flip inelastic tunneling via a thermally excited magnon, i.e., the Zhang model with an evidence of the role played by magnons indicated by tunneling spectroscopy $[51,52,56]$. Alternatively, the increase in $G_{\mathrm{P}}$ with $T$ for an epitaxial MTJ has been explained within the framework of the Shang model by taking into account the smearing effect of the Fermi distribution function at finite temperatures [53], which increases $G$ as $T$ increases [61]. Note, however, that the influence of spin-flip inelastic tunneling was ignored in the Shang-model-based analysis. On the other hand, the origin of the decrease in $G_{\mathrm{P}}$ with increasing $T$ observed for some epitaxial MTJs [58-60] is not yet fully understood. Thus, a more systematic experimental study is essential to get a deeper understanding of the origin of the $T$ dependence of spin-dependent $G_{\mathrm{P}}$ and $G_{\mathrm{AP}}$.

A promising approach would be to investigate half-metalbased MTJs with systematically varied $P$ values at $0 \mathrm{~K}$, as MTJs with highly spin-polarized electrodes arising from half-metallicity would show most typical $T$ dependence of $G_{\mathrm{P}}$ and $G_{\mathrm{AP}}$. In addition, it is a particularly desirable approach to extract how the $T$ dependence of $G_{\mathrm{P}}$ and $G_{\mathrm{AP}}$ changes with $P$ at $0 \mathrm{~K}$ by investigating a series of high-quality MTJs with systematically varied $P$ at $0 \mathrm{~K}$. Furthermore, development of a more comprehensive model for the $T$ dependence of $G_{\mathrm{P}}$ and $G_{\mathrm{AP}}$ is requisite as the predictions of the Zhang and Shang models for $G_{\mathrm{P}}(T)$ are opposite. This difference in the predictions for $G_{\mathrm{P}}(T)$ also suggests that an analysis of the experimental $G_{\mathrm{P}}(T)$ would be critical to revealing the origin of the $T$ dependence of spin-dependent $G$.

The purpose of this paper is to elucidate the origin of the $T$ dependence of $G_{\mathrm{P}}$ and $G_{\mathrm{AP}}$ of MTJs through analyzing the experimental $G_{\mathrm{P}}(T)$ and $G_{\mathrm{AP}}(T)$ of high-quality MTJs showing giant TMRs and to provide a unified picture of how the $T$ dependence of $G_{\mathrm{P}}$ and $G_{\mathrm{AP}}$ changes with $P$. To do so, we experimentally investigated the $T$ dependences of $G_{\mathrm{P}}$ and $G_{\mathrm{AP}}$ of CMS MTJs having systematically varied spin polarizations at $4.2 \mathrm{~K}$ by varying the Mn composition $\alpha$ in $\mathrm{Co}_{2} \mathrm{Mn}_{\alpha} \mathrm{Si}$ electrodes that exhibited a giant TMR ratio of up to $2110 \%$ at $4.2 \mathrm{~K}$ (up to $366 \%$ at $290 \mathrm{~K}$ ) $[11,16]$. Notable features in $G_{\mathrm{P}}(T)$ were found; i.e., it decreased with increasing $T$ from $T_{1}$ of about $30 \mathrm{~K}$ to $T_{2}$ ranging from about $162 \mathrm{~K}$ to $237 \mathrm{~K}$ depending on $\alpha$; then it increased for $T>T_{2}$. Furthermore, as $P$ at $4.2 \mathrm{~K}$ increased, $T_{2}$ increased, and the maximum decrease in the normalized $G_{\mathrm{P}}\left[=G_{\mathrm{P}}(T) / G_{\mathrm{P}}(4.2 \mathrm{~K})\right]$ at $T_{2}$ increased. To clarify the origin of the characteristic $T$ dependence of $G_{\mathrm{P}}$, we developed a model that took into account both spin-conserving elastic tunneling in which $P$ decays with increasing $T$ and spin-flip inelastic tunneling via a magnon by extending the original Zhang model [49]. The extension of the Zhang model was possible because of its generality in the sense that its expressions of $G_{\mathrm{P}}(T)$ and $G_{\mathrm{AP}}(T)$ include both elastic and inelastic tunneling terms, although $P$ is assumed to be independent of $T$. Accordingly, we showed that the proposed model could reproduce the nonmonotonic behavior of the experimental $G_{\mathrm{P}}(T)$. Furthermore, the dependence of the experimental $G_{\mathrm{P}}(T)$ on $P$ at $4.2 \mathrm{~K}$ was well explained by the proposed model. The model also consistently explained the experimental $G_{\mathrm{AP}}(T)$, including a stronger $T$ dependence for higher $P$ at $4.2 \mathrm{~K}$. Thus, it was found that elastic tunneling with decaying $P$ with increasing $T$ and inelastic tunneling via a magnon are two key factors that determine the $T$ dependence of spin-dependent tunneling conductance of MTJs. This finding is generally applicable to MTJs with a wide range of $P$. It was also indicated that an analysis of the experimental $G_{\mathrm{P}}(T)$ is critical to revealing the origin of the $T$ dependence of $G_{\mathrm{P}}$ and $G_{\mathrm{AP}}$.

The paper is organized as follows. Section II presents our experimental methods. Section III presents the results and discussion. Section III A first describes the overall features of $G_{\mathrm{P}}(T)$ and $G_{\mathrm{AP}}(T)$ of CMS MTJs with various $\alpha$ values that showed a high TMR ratio of up to $2110 \%$ at $4.2 \mathrm{~K}$ $(366 \%$ at $290 \mathrm{~K})$. Then it describes $G_{\mathrm{P}}(T)$ in detail. After that, it describes the extension of the Zhang model that incorporates a $T$-dependent $P$ to explain the characteristic $T$ dependence of $G_{\mathrm{P}}$ observed for the CMS MTJs with half-metallic electrodes. Section III B shows that $G_{\mathrm{AP}}(T)$ can be also consistently explained by this extended Zhang model. Section IV summarizes our results and concludes the paper. 


\section{EXPERIMENTAL METHODS}

We measured and analyzed the $G_{\mathrm{P}}(T)$ and $G_{\mathrm{AP}}(T)$ mainly of two series of $\mathrm{CO}_{50} \mathrm{Fe}_{50}$ ( $\mathrm{CoFe}$ )-buffered CMS MTJs having $\mathrm{Co}_{2} \mathrm{Mn}_{\alpha} \mathrm{Si}_{0.84}$ electrodes or $\mathrm{Co}_{2} \mathrm{Mn}_{\alpha} \mathrm{Si}_{0.96}$ electrodes. The series had various $\alpha$ values ranging from a Mn-deficient composition of $\alpha<2-\beta$, where $\beta$ is the Si composition in the film composition expression of $\mathrm{Co}_{2} \mathrm{Mn}_{\alpha} \mathrm{Si}_{\beta}$, to a Mn-rich one of $\alpha>2-\beta$. The MTJ layer structure was as follows: (from the substrate side) $\mathrm{MgO}$ buffer $(10 \mathrm{~nm}) / \mathrm{CoFe}$ buffer (30 $\mathrm{nm}) / \mathrm{CMS}$ lower electrode $(3 \mathrm{~nm}) / \mathrm{MgO}$ barrier $(1.4-$ $3.2 \mathrm{~nm}) / \mathrm{CMS}$ upper electrode $(3 \mathrm{~nm}) / \mathrm{CoFe}(1.1 \mathrm{~nm}) / \mathrm{Ir}_{22} \mathrm{Mn}_{78}$ $(10 \mathrm{~nm}) / \mathrm{Ru}$ cap $(5 \mathrm{~nm})$, grown on a $\mathrm{MgO}(001)$ substrate, as shown in Fig. 1. The fabrication procedure of the CoFebuffered CMS MTJs has been reported elsewhere [11]. Briefly, each layer was successively deposited in an ultrahigh-vacuum chamber equipped with magnetron cathodes and an electronbeam evaporator. The CMS electrodes were prepared by magnetron cosputtering from a nearly stoichiometric CMS target and $\mathrm{Mn}$ target (with a base pressure of about $6 \times 10^{-8} \mathrm{~Pa}$ ). The $\mathrm{MgO}$ tunnel barrier in the MTJ layer structure had a wedge shape, and its nominal thickness was varied from 1.4 to $3.2 \mathrm{~nm}$ on each $20 \times 20 \mathrm{~mm}^{2} \mathrm{MgO}(001)$ substrate by linearly moving the shutter during the deposition by electron-beam evaporation. Figure 2 plots typical dependence on $t_{\mathrm{MgO}}$ of $R_{\mathrm{P}} A, R_{\mathrm{AP}} A$, and the TMR ratio at $290 \mathrm{~K}$ for CMS MTJs with $\mathrm{Co}_{2} \mathrm{Mn}_{1.30} \mathrm{Si}_{0.84}$ electrodes fabricated on a $20 \times 20 \mathrm{~mm}^{2}$ substrate over a $t_{\mathrm{MgO}}$ range from 2.2 to $3.1 \mathrm{~nm}$, where $A$ is the nominal junction area of $10 \times 10 \mu \mathrm{m}^{2}$. We have reported TMR ratios of up to $2110 \%$ at $4.2 \mathrm{~K}$ and $366 \%$ at $290 \mathrm{~K}$ for these MTJs [16]. The dependences of $R_{\mathrm{P}} A$ and $R_{\mathrm{AP}} A$ on $t_{\mathrm{MgO}}$ were exponential with almost identical slopes, resulting in nearly constant TMR ratios from 2.2 to $3.1 \mathrm{~nm}$ of $t_{\mathrm{MgO}}$. Furthermore, TMR ratios over $350 \%$ were obtained for a wide $t_{\mathrm{MgO}}$ range from 2.3 to $3.0 \mathrm{~nm}$, with the maximum TMR ratio being

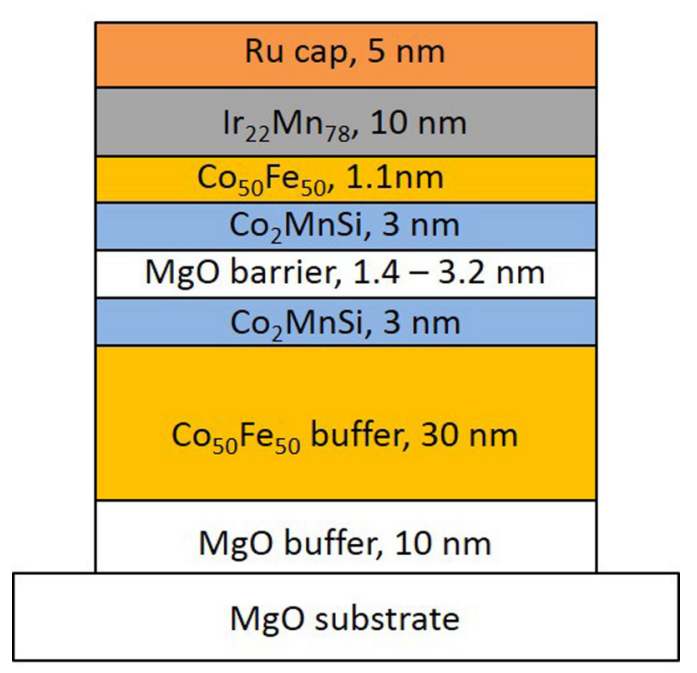

FIG. 1. Schematic diagram of the layer structure of $\mathrm{Co}_{50} \mathrm{Fe}_{50}$ (CoFe)-buffered $\mathrm{Co}_{2} \mathrm{Mn}_{\alpha} \mathrm{Si}_{\beta}$ (CMS)/MgO/CMS MTJ (CMS MTJ) consisting of (from the substrate side) $\mathrm{MgO}$ buffer $(10 \mathrm{~nm}) / \mathrm{CoFe}$ buffer $(30 \mathrm{~nm}) / \mathrm{CMS}$ lower electrode $(3 \mathrm{~nm}) / \mathrm{MgO}$ barrier (1.4$3.2 \mathrm{~nm}) / \mathrm{CMS}$ upper electrode $(3 \mathrm{~nm}) / \mathrm{CoFe}(1.1 \mathrm{~nm}) / \mathrm{Ir}_{22} \mathrm{Mn}_{78}$ $(10 \mathrm{~nm}) / \mathrm{Ru}$ cap $(5 \mathrm{~nm})$, grown on a $\mathrm{MgO}(001)$ single-crystal substrate.

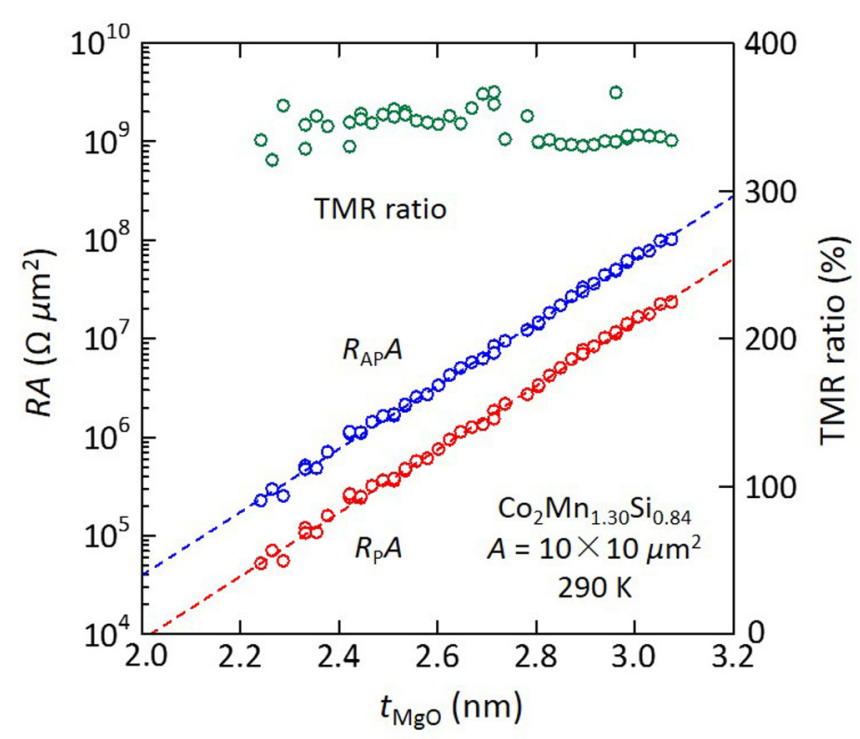

FIG. 2. Typical dependence of $R_{\mathrm{P}} A, R_{\mathrm{AP}} A$, and TMR ratio on $\mathrm{MgO}$ barrier thickness $\left(t_{\mathrm{MgO}}\right)$ at $290 \mathrm{~K}$ for CMS MTJs with $\mathrm{Co}_{2} \mathrm{Mn}_{1.30} \mathrm{Si}_{0.84}$ electrodes fabricated on a $20 \times 20 \mathrm{~mm}^{2}$ substrate over a $t_{\mathrm{MgO}}$ range from 2.2 to $3.1 \mathrm{~nm}$, where $A$ is the nominal junction area of $10 \times 10 \mu \mathrm{m}^{2}$. The $\mathrm{MgO}$ tunnel barrier had a wedge structure, and its nominal thickness was varied from 1.4 to $3.2 \mathrm{~nm}$ on each $20 \times 20 \mathrm{~mm}^{2}$ substrate by linearly moving the shutter during the deposition by electron-beam evaporation.

$366 \%$ at $290 \mathrm{~K}$. The value of $m^{*} \varphi_{0}(0.51 \mathrm{eV})$ was determined from the slope of the $\ln \left(R_{\mathrm{P}} A\right)$ versus $t_{\mathrm{MgO}}$, where $m^{*}$ is the effective mass normalized by the electron rest mass and $\varphi_{0}$ is the potential barrier height (the energy difference between the $E_{\mathrm{F}}$ of the emitter ferromagnetic electrode and the bottom of the conduction band in the tunnel barrier). This value was close to that of $0.32 \mathrm{eV}$ for epitaxial $\mathrm{Co}_{2} \mathrm{Cr}_{0.6} \mathrm{Fe}_{0.4} \mathrm{Al} / \mathrm{MgO} / \mathrm{CoFe}$ MTJs [62] and $0.39 \mathrm{eV}$ for epitaxial $\mathrm{Fe} / \mathrm{MgO} / \mathrm{Fe}$ MTJs [63].

The tunneling conductances $G_{\mathrm{P}}(T)$ and $G_{\mathrm{AP}}(T)$ were measured using a dc four-probe method at temperatures from 4.2 to $290 \mathrm{~K}$ at both positive and negative bias voltages of $\pm 2 \mathrm{mV}$. Both polarities for the bias voltage were used to cancel the small thermoelectric force, which is particularly important for accurately measuring $G_{\mathrm{P}}$ values. The TMR ratio was defined as TMR $=\left(G_{\mathrm{P}}-G_{\mathrm{AP}}\right) / G_{\mathrm{AP}}=\left(R_{\mathrm{AP}}-R_{\mathrm{P}}\right) / R_{\mathrm{P}}$, where $G_{\mathrm{P}}\left(R_{\mathrm{P}}\right)$ and $G_{\mathrm{AP}}\left(R_{\mathrm{AP}}\right)$ are the tunneling conductances (resistances) for the parallel and antiparallel magnetization alignments between the upper and lower electrodes.

\section{RESULTS AND DISCUSSION}

\section{A. $T$ dependence of tunneling conductance for the parallel alignment}

In this section, we first describe the overall features of the experimental $T$ dependences of $G_{\mathrm{P}}$ and $G_{\mathrm{AP}}$ of CoFe-buffered CMS MTJs with $\mathrm{Co}_{2} \mathrm{Mn}_{\alpha} \mathrm{Si}_{0.84}$ electrodes with various $\alpha$ values ranging from a Mn-deficient $\alpha=0.73$ to Mn-rich $\alpha=1.24$ and 1.30. Then we describe the features of the experimental $G_{\mathrm{P}}(T)$ in detail. Given the experimental results, we devise an extension of the Zhang model by introducing a $T$-dependent spin polarization and use it to discuss the origin 

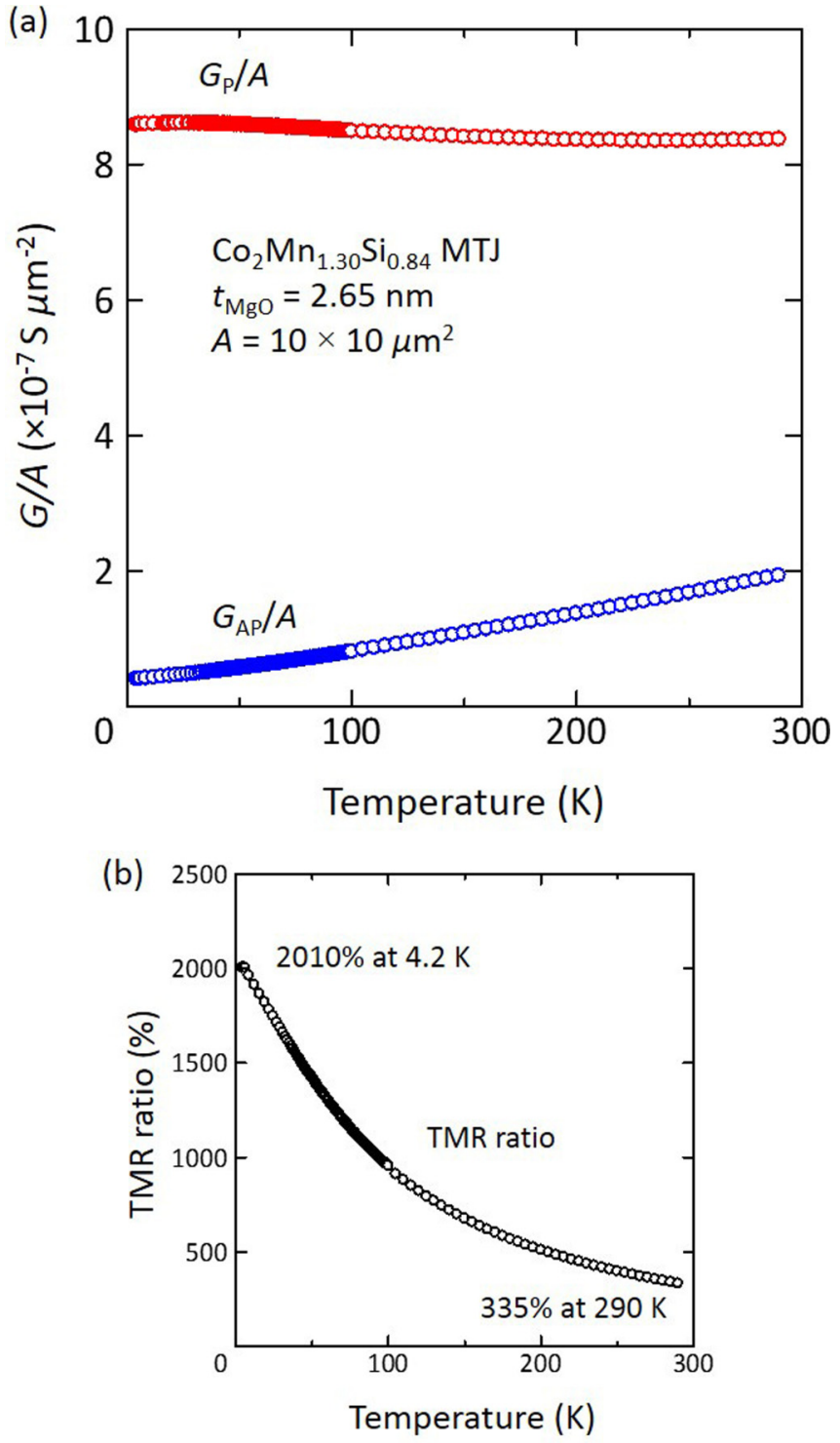

FIG. 3. (a) Typical temperature dependence of $G_{\mathrm{P}} / A$ and $G_{\mathrm{AP}} / A$ of a CMS MTJ with Mn-rich $\mathrm{Co}_{2} \mathrm{Mn}_{1.30} \mathrm{Si}_{0.84}$ electrodes and $t_{\mathrm{MgO}}=$ $2.65 \mathrm{~nm}$ from 4.2 to $290 \mathrm{~K}$ showing TMR ratios of $2010 \%$ at $4.2 \mathrm{~K}$ and $335 \%$ at $290 \mathrm{~K}$, where $G_{\mathrm{P}}$ and $G_{\mathrm{AP}}$ are the tunneling conductances for the parallel and antiparallel magnetization alignments between the lower and upper electrodes, and $A$ is the nominal junction area of $10 \times 10 \mu \mathrm{m}^{2}$. The bias voltage was $2 \mathrm{mV}$. (b) Resulting $T$ dependence of the TMR ratio.

of the observed $T$ dependence of $G_{\mathrm{P}}$ through analyzing the experimental $G_{\mathrm{P}}(T)$. The $\mathrm{Co}_{2} \mathrm{Mn}_{\alpha} \mathrm{Si}_{0.96}$ MTJs with various $\alpha$ showed almost identical $T$ dependences for $G_{\mathrm{P}}$ and $G_{\mathrm{AP}}$. The $\alpha$ dependence of the TMR ratio of the $\mathrm{Co}_{2} \mathrm{Mn}_{\alpha} \mathrm{Si}_{0.84}$ MTJ series has been reported, and a TMR ratio of up to $2110 \%$ at $4.2 \mathrm{~K}(366 \%$ at $290 \mathrm{~K})$ has been demonstrated for this MTJ series [16]. Figure 3(a) shows typical $T$ dependence of $G_{\mathrm{P}}$ and $G_{\mathrm{AP}}$ from 4.2 to $290 \mathrm{~K}$ of a CMS MTJ with $\alpha=1.30\left(\mathrm{Co}_{2} \mathrm{Mn}_{1.30} \mathrm{Si}_{0.84}\right.$ electrodes $)$ that showed TMR ratios of $2010 \%$ at $4.2 \mathrm{~K}$ and $335 \%$ at $290 \mathrm{~K}$. Here, $G_{\mathrm{AP}}$ showed a strong $T$ dependence $\left(G_{\mathrm{AP}}\right.$ at $290 \mathrm{~K}$ was 4.6 times higher than that at $4.2 \mathrm{~K}$ ), while $G_{\mathrm{P}}$ changed only about
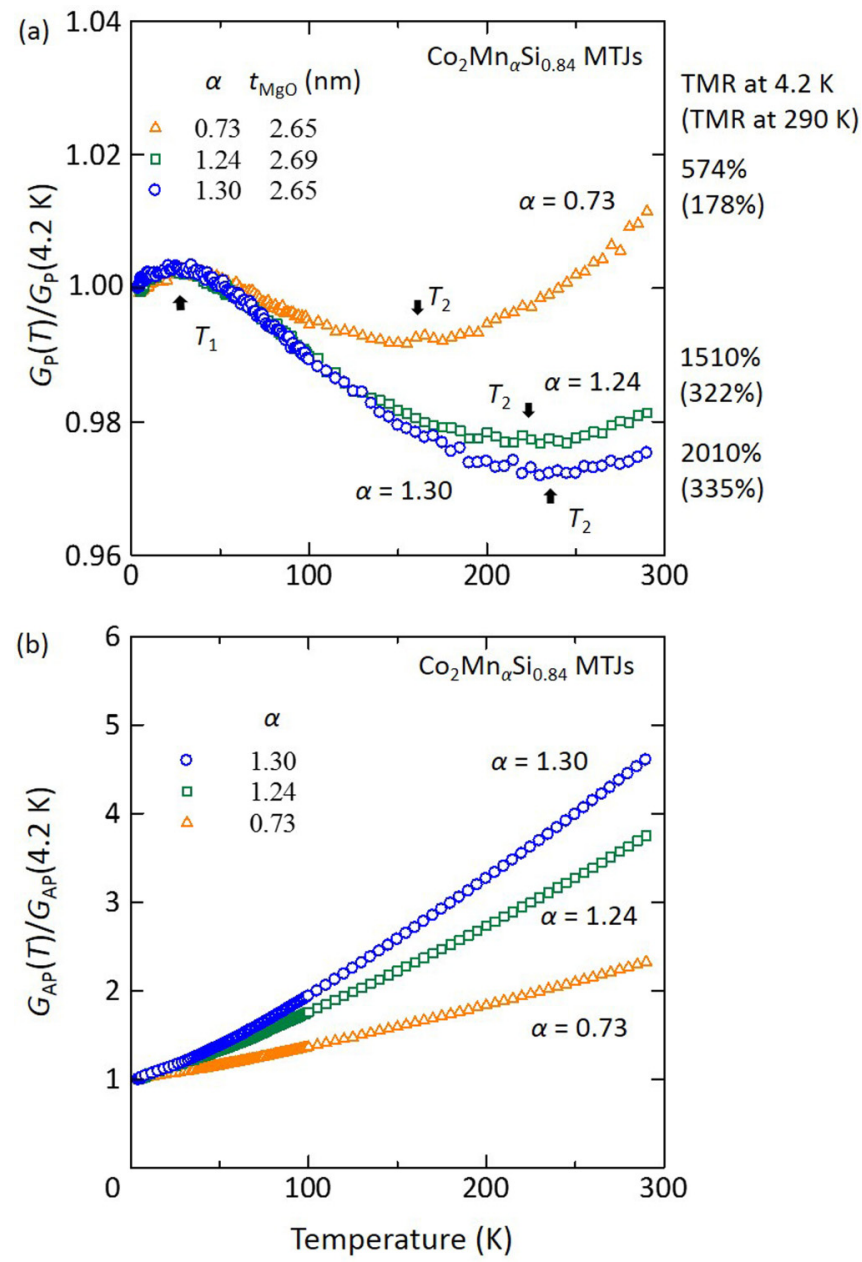

FIG. 4. (a) Normalized $G_{\mathrm{P}}$ and (b) $G_{\mathrm{AP}}$ as a function of $T$ from 4.2 to $290 \mathrm{~K}$ for CMS MTJs with various $\alpha$ ranging from Mn-deficient $\alpha=0.73$ to Mn-rich $\alpha=1.30$ in $\mathrm{Co}_{2} \mathrm{Mn}_{\alpha} \mathrm{Si}_{0.84}$ electrodes, where $G_{\mathrm{P}}$ and $G_{\mathrm{AP}}$ are normalized by the respective values at $4.2 \mathrm{~K}$. The data of $\alpha=1.30 \mathrm{MTJ}$ are the same as those shown in Fig. 3 .

$3 \%$ and could be regarded as being almost independent of $T$ compared with the strong variation in $G_{\mathrm{AP}}$. Because of these characteristics, the $T$ dependence of the TMR ratio was determined mostly by that of $G_{\mathrm{AP}}$, resulting in a strong $T$ dependence of the TMR ratio [Fig. 3(b)]. Figure 4(a) plots the experimental $G_{\mathrm{P}}(T)$ normalized by the respective values at $4.2 \mathrm{~K}, G_{\mathrm{P}, \mathrm{N}}(T)=G_{\mathrm{P}}(T) / G_{\mathrm{P}}(4.2 \mathrm{~K})$, of the CMS MTJs with various $\alpha$ values. Furthermore, Fig. 4(b) plots the experimental $G_{\mathrm{AP}}(T)$ normalized by the respective values at $4.2 \mathrm{~K}, G_{\mathrm{AP}, \mathrm{N}}(T)=G_{\mathrm{AP}}(T) / G_{\mathrm{AP}}(4.2 \mathrm{~K})$. The TMR ratio at $4.2 \mathrm{~K}$ of these CMS MTJs increased with increasing $\alpha$ from 0.73 to 1.30 (Table I). The increase in the TMR ratio has been explained by the enhancement of half-metallicity due to the suppression of $\mathrm{Co}_{\mathrm{Mn}}$ antisites for a $\mathrm{Mn}$-rich composition $[10,12]$. As a basis for the characterization and analysis of the experimental $G_{\mathrm{P}}(T)$ and $G_{\mathrm{PP}}(T)$, we deduced the spin polarization at $4.2 \mathrm{~K}, P(4.2 \mathrm{~K})$, for these MTJs from the TMR ratios at $4.2 \mathrm{~K}$ by assuming the Julliere model [64], i.e.,

$$
\text { TMR ratio }=\frac{2 P^{2}}{1-P^{2}},
$$


TABLE I. TMR ratios at 4.2 and $290 \mathrm{~K}$ of CMS MTJs with various $\alpha$ in $\mathrm{Co}_{2} \mathrm{Mn}_{\alpha} \mathrm{Si}_{0.84}$ electrodes of which $G_{\mathrm{P}, \mathrm{N}}(T)$ and $G_{\mathrm{AP}, \mathrm{N}}(T)$ are shown in Fig. 4 along with the respective nominal $\mathrm{MgO}$ barrier thicknesses $\left(t_{\mathrm{MgO}}\right)$. The tunneling spin polarizations at $4.2 \mathrm{~K}, P(4.2 \mathrm{~K})$, deduced from the Julliere model [Eq. (1)] with the respective TMR ratios at $4.2 \mathrm{~K}$, are also shown. The tunneling spin polarizations at $0 \mathrm{~K}, P_{0}$, could be approximated by the respective $P(4.2 \mathrm{~K})$ and could be used in the analysis.

\begin{tabular}{lcccc}
\hline \hline$\alpha$ in $\mathrm{Co}_{2} \mathrm{Mn}_{\alpha} \mathrm{Si}_{0.84}$ & $t_{\mathrm{MgO}}(\mathrm{nm})$ & TMR ratio at $4.2 \mathrm{~K}$ & TMR ratio at $290 \mathrm{~K}$ & $P(4.2 \mathrm{~K})$ \\
\hline 0.73 & 2.65 & $574 \%$ & $178 \%$ & 0.861 \\
1.24 & 2.69 & $1510 \%$ & $322 \%$ & 0.940 \\
1.30 & 2.65 & $2010 \%$ & $335 \%$ & 0.954 \\
\hline \hline
\end{tabular}

where $P$ is defined using the majority-spin and minorityspin density of states (DOS) at $E_{\mathrm{F}}, \rho_{\mathrm{M}}$ and $\rho_{\mathrm{m}}$, as $P=$ $\left(\rho_{\mathrm{M}}-\rho_{\mathrm{m}}\right) /\left(\rho_{\mathrm{M}}+\rho_{\mathrm{m}}\right)$. We also assumed that the lower and upper electrodes had an identical $P$. Table I summarizes the deduced $P(4.2 \mathrm{~K})$ for the CMS MTJs. As demonstrated by their high TMR ratios at $4.2 \mathrm{~K}$, the CMS MTJs exhibited significantly high $P$ values of up to 0.954 at $4.2 \mathrm{~K}$ corresponding to the experimentally obtained TMR ratio of $2010 \%$ at $4.2 \mathrm{~K}$. We approximated $P$ at $0 \mathrm{~K}, P_{0}$, by $P(4.2 \mathrm{~K})$ in the following analysis. The crucial role of coherent tunneling $[65,66]$ has been experimentally [11] and theoretically [67] demonstrated in CMS MTJs. Thus, the $P$ deduced from Eq. (1) is the value determined by $\rho_{\mathrm{M}}$ and $\rho_{\mathrm{m}}$ arising from the electronic states contributing to coherent tunneling [12,17]. In this sense, the deduced $P$ should be called the tunneling spin polarization, although we will still refer to it as the tunneling spin polarization or spin polarization without distinguishing the meanings.

Note that the normalized $G_{\mathrm{P}}(T)$ and $G_{\mathrm{AP}}(T)$ data plotted in Fig. 4 are for MTJs having almost identical nominal $t_{\mathrm{MgO}}$ values of $2.66 \mathrm{~nm} \pm 1 \%$ (Table I). Thus, the smearing effect of the Fermi distribution function at finite temperatures to the tunneling conductance $[50,52,61]$ can be regarded as almost identical for these MTJs.

As shown in Fig. 4(a), the $G_{\mathrm{P}, \mathrm{N}}(T)$ of these MTJs exhibited distinct features, even though it can be regarded to be almost independent of $T$ compared with the strong $T$ dependence of $G_{\mathrm{AP}, \mathrm{N}}(T)$. The first feature is that the $G_{\mathrm{P}, \mathrm{N}}$ decreased with increasing $T$ from $T=T_{1}$ of about $30 \mathrm{~K}$ to $T=T_{2}$ ranging from about $162 \mathrm{~K}$ for $\alpha=0.73$ to $237 \mathrm{~K}$ for $\alpha=1.30$.
Then it increased for $T>T_{2}$. The second feature is that $T_{2}$ increased as the TMR ratio at $4.2 \mathrm{~K}$ increased or equivalently $P(4.2 \mathrm{~K})$ increased, as shown in Fig. 5(a). The third feature is that the maximum decrease in the experimental $G_{\mathrm{P}, \mathrm{N}}$ at $T_{2}$, defined as $\Delta G_{\mathrm{P}, \mathrm{N}}\left(T_{2}\right)=G_{\mathrm{P}, \mathrm{N}}\left(T_{2}\right)-1$ increased in magnitude as $P(4.2 \mathrm{~K})$ increased, as shown in Fig. 5(b).

On the other hand, the degree of the $T$ dependence of $G_{\mathrm{AP}, \mathrm{N}}$ increased as $P(4.2 \mathrm{~K})$ increased. Our previous paper discussed the $T$ dependence of $G_{\mathrm{AP}}$ for CMS MTJs with various tunneling spin polarizations in terms of $G_{\mathrm{AP}}(290 \mathrm{~K}) / G_{\mathrm{AP}}(4.2 \mathrm{~K})$ according to the original Zhang model [12]. This paper discusses the key factors that determine the $T$ dependences of $G_{\mathrm{P}}$ and $G_{\mathrm{AP}}$ from a wider point of view.

Now let us discuss how these features of $G_{\mathrm{P}, \mathrm{N}}(T)$ and $G_{\mathrm{AP}, \mathrm{N}}(T)$ can be explained consistently. We will first analyze the experimental $T$ dependence of $G_{\mathrm{P}, \mathrm{N}}$. The comparison of the experimental $G_{\mathrm{P}, \mathrm{N}}(T)$ with the predictions clearly indicates that neither the Shang model nor the Zhang model can explain the experimental $G_{\mathrm{P}, \mathrm{N}}(T)$. Thus, an analysis of $G_{\mathrm{P}, \mathrm{N}}(T)$ is crucial to clarifying the origin of the $T$ dependence of spin-dependent tunneling conductance in MTJs with highly spin-polarized electrodes. We will focus on the decrease in $G_{\mathrm{P}, \mathrm{N}}$ as $T$ increases from $T_{1}$ to $T_{2}$ and the increase in $G_{\mathrm{P}, \mathrm{N}}$ as $T$ increases for $T>T_{2}$. Furthermore, taking into consideration that the variation in $G_{\mathrm{P}, \mathrm{N}}$ for $T<T_{1}$ was smaller than that for $T>T_{1}$ by one order of magnitude, we will ignore the small increase in $G_{\mathrm{P}}$ for $4.2 \mathrm{~K}<T<T_{1}$. Regarding the experimental $G_{\mathrm{P}, \mathrm{N}}(T)$, the prediction of the Shang model for $G_{\mathrm{P}}$, i.e., the negative $d G_{\mathrm{P}} / d T$, is qualitatively in agreement with the experimental decrease in $G_{\mathrm{P}}$ for the $T$ range from $T_{1}$ to
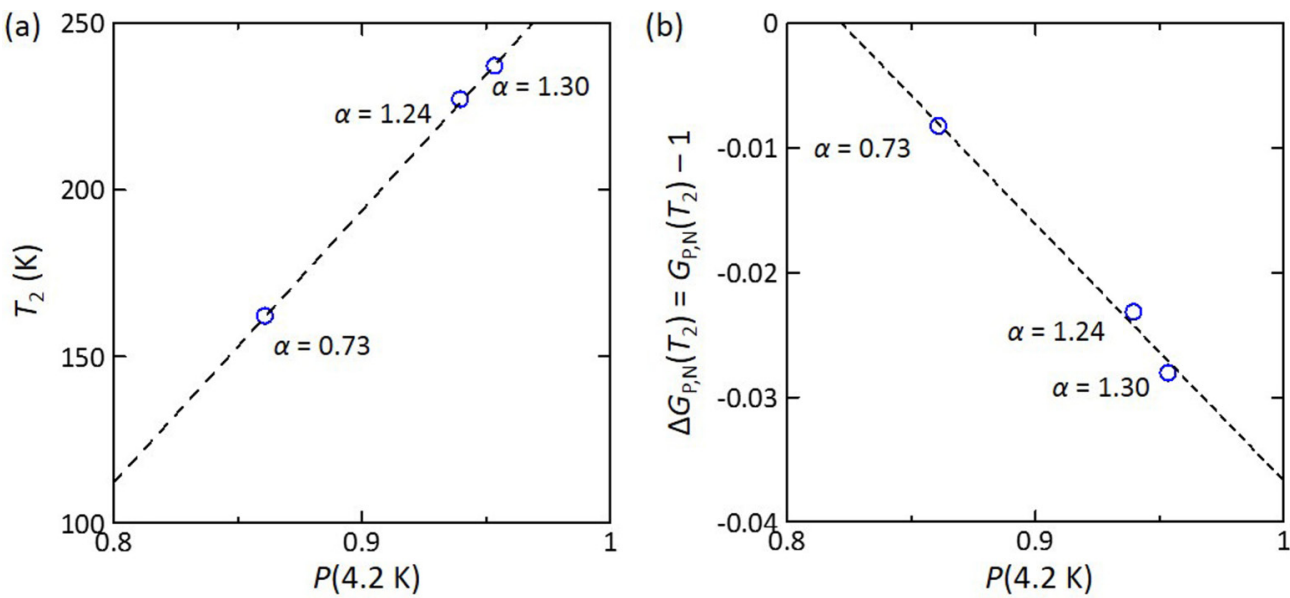

FIG. 5. Dependence of (a) the characteristic temperature $T_{2}$ and (b) the maximum decrease in the normalized $G_{\mathrm{P}}\left(G_{\mathrm{P}, \mathrm{N}}\right)$ at $T_{2}$, defined as $\Delta G_{\mathrm{P}, \mathrm{N}}\left(T_{2}\right)=G_{\mathrm{P}, \mathrm{N}}\left(T_{2}\right)-1$, on the tunneling spin polarization at $4.2 \mathrm{~K}, P(4.2 \mathrm{~K})$. The straight lines are guides for the eye. 
$T_{2}$, whereas the prediction of the Zhang model, i.e., the positive $d G_{\mathrm{P}} / d T$, is qualitatively in agreement with the experimental increase in $G_{\mathrm{P}}$ in the $T$ range above $T_{2}$. Furthermore, the original Zhang model is more general than the Shang model in the sense that its expression for the tunneling conductance consists of both a spin-conserving elastic tunneling term and a spin-flip inelastic tunneling term, while the Shang expression consists only of a spin-conserving elastic tunneling term. Given its greater generality, we decided to extend the original Zhang model by introducing a $T$ dependence of $P$, i.e., a $T$ dependence of $\rho_{\mathrm{M}}$ and $\rho_{\mathrm{m}}$. This extension leads to a $T$ dependence in the elastic tunneling term. We also took into consideration the effect of the broadening of the Fermi distribution, i.e., the smearing effect [50,52,61], on $G_{\mathrm{P}}(T)$. In such a case, the elastic and inelastic tunneling conductance terms for the parallel alignment at a finite $T$ are multiplied by a factor

$$
S_{\mathrm{e}}(T)=C T / \sin (C T),
$$

where $C=1.387 \times 10^{-3} t / \varphi^{1 / 2}, t$ is the thickness of the barrier (in nanometers), and $\varphi$ is the barrier height (in electronvolts) [61]. We assumed a $\varphi$ of $3.5 \mathrm{eV}$, which corresponds to half the $\mathrm{MgO}$ band gap [52]. The validity of this value will be described later in this section. A typical value of $S_{\mathrm{e}}(T)$ at $290 \mathrm{~K}$ is 1.056 for a $t_{\mathrm{MgO}}$ of $2.65 \mathrm{~nm}$. The introduction of this small factor is required to fit the $G_{\mathrm{P}}(T)$ of the CMS MTJs that had high spin polarization. This is because its variation as a function of $T$ became small, i.e., being comparable to the variation in $S_{\mathrm{e}}(T)$ as $P_{0}$ increases. Accordingly, $G_{\mathrm{P}}(T)$ in the extended Zhang model for $k_{\mathrm{B}} T>E_{\mathrm{c}}$ is

$$
\begin{aligned}
G_{\mathrm{P}}(T) & =G_{\mathrm{P}, \mathrm{el}}(T)+G_{\mathrm{P}, \text { in }}(T), \\
G_{\mathrm{P}, \mathrm{el}}(T) & =S_{\mathrm{e}}(T) Q_{1}\left\{\left[\rho_{\mathrm{M}}(T)\right]^{2}+\left[\rho_{\mathrm{m}}(T)\right]^{2}\right\}, \\
G_{\mathrm{P}, \text { in }}(T) & =S_{\mathrm{e}}(T) Q_{2} k_{\mathrm{B}} T \cdot f(T) \cdot 2 \rho_{\mathrm{M}}(T) \rho_{\mathrm{m}}(T),
\end{aligned}
$$

where $Q_{1}, Q_{2}$, and $f(T)$ are

$$
\begin{aligned}
Q_{1} & =\frac{4 \pi e^{2}}{\hbar}\left(\left|T^{d}\right|^{2}+2 S^{2}\left|T^{J}\right|^{2}\right), \\
Q_{2} & =\frac{4 \pi e^{2}}{\hbar} \frac{2 S}{E_{\mathrm{m}}}\left|T^{J}\right|^{2}, \\
f(T) & =-\ln \left[1-\exp \left(-\frac{E_{\mathrm{c}}}{k_{\mathrm{B}} T}\right)\right] .
\end{aligned}
$$

The definitions of the parameters $T^{d}, T^{J}, S, E_{\mathrm{m}}$, and $E_{\mathrm{c}}$ are given elsewhere [49]. Briefly, $T^{d}$ and $T^{J}$ are tunneling matrix elements for direct tunneling and for tunneling under the $s$ - $d$ exchange interaction between local and itinerant electrons at the interface, respectively, $S$ is the spin parameter, $E_{\mathrm{m}}=3 k_{\mathrm{B}} T_{\mathrm{C}} /(S+1), E_{\mathrm{c}}$ is the lower magnon cutoff energy, $k_{\mathrm{B}}$ is the Boltzmann constant, and $T_{\mathrm{C}}$ is the Curie temperature of the ferromagnetic electrode. The only difference between this and the original model is that $\rho_{\mathrm{M}}$ and $\rho_{\mathrm{m}}$ are dependent on $T$ in the former, and they are independent of $T$ in the latter. The first term of Eq. (3a), $G_{\mathrm{P}, \mathrm{el}}(T)$, represents the spin-conserving elastic tunneling process, and the second term, $G_{\mathrm{P}, \text { in }}(T)$, represents the spin-flip inelastic tunneling process via a thermally excited magnon. The first term is equivalent to the Shang model, and also to the Julliere model, although the latter does not consider the $T$ dependence of $P$. Here, $G_{\mathrm{P}}$ at $T=0$ consists of spin-conserving elastic tunneling, which from Eq. (3) can be expressed as

$$
G_{\mathrm{P}}(T=0)=Q_{1}\left\{\left[\rho_{\mathrm{M}}(T=0)\right]^{2}+\left[\rho_{\mathrm{m}}(T=0)\right]^{2}\right\},
$$

[note $S_{\mathrm{e}}(T=0)=1$ ]. Thus, $G_{\mathrm{P}}(T)$ normalized by its value at $T=0, G_{\mathrm{P}, \mathrm{N}}(T)$, is

$$
\begin{aligned}
G_{\mathrm{P}, \mathrm{N}}(T)= & \frac{G_{\mathrm{P}}(T)}{G_{\mathrm{P}}(T=0)} \\
= & S_{\mathrm{e}}(T)\left\{1-\frac{P_{0}^{2}-[P(T)]^{2}}{1+P_{0}^{2}}\right\} \\
& +S_{\mathrm{e}}(T) a\left\{\frac{1-[P(T)]^{2}}{1+P_{0}^{2}}\right\} k_{\mathrm{B}} T \cdot f(T),
\end{aligned}
$$

where $P(T)$ is the spin polarization at $T$, defined as

$$
\begin{aligned}
P(T) & =\frac{\rho_{\mathrm{M}}(T)-\rho_{\mathrm{m}}(T)}{\rho_{\mathrm{M}}(T)+\rho_{\mathrm{m}}(T)}, \\
a & =Q \frac{2 S}{E_{\mathrm{m}}}, \\
Q & =\frac{1}{\frac{\left|T^{d}\right|^{2}}{\left|T^{J}\right|^{2}}+2 S^{2}},
\end{aligned}
$$

and the relations $\left(\rho_{\mathrm{M}}\right)^{2}+\left(\rho_{\mathrm{m}}\right)^{2}=(1 / 2)\left(\rho_{\mathrm{M}}+\rho_{\mathrm{m}}\right)^{2}\left(1+P^{2}\right)$ and $2 \rho_{\mathrm{M}} \rho_{\mathrm{m}}=(1 / 2)\left(\rho_{\mathrm{M}}+\rho_{\mathrm{m}}\right)^{2}\left(1-P^{2}\right)$ are used along with an assumption of $\left(\rho_{\mathrm{M}}+\rho_{\mathrm{m}}\right)$ being independent of $T$. To fit the experimental $G_{\mathrm{P}}(T)$, we introduced the following Bloch type $T^{3 / 2}$ dependence for $P$ as in the Shang model [50]:

$$
P(T)=P_{0}\left(1-\eta T^{3 / 2}\right),
$$

where $\eta$ is a material-dependent constant. Note that Eq. (5) does not include a $T$-dependent term to explain the small decrease in $G_{\mathrm{P}}$ as $T$ decreases from $T_{1}$ of about $30 \mathrm{~K}$ to $4.2 \mathrm{~K}$. Thus, the fitted curve for a $T$ range below $T_{1}$ becomes extrapolated from a $T$ range above $T_{1}$. Because of this disagreement between the experimental and calculated $G_{\mathrm{P}, \mathrm{N}}$ for $4.2 \mathrm{~K}<T<T_{1}$, a small constant $c_{0}$ was added to Eq. (5) for fitting. The final form of the normalized $G_{\mathrm{P}}(T)$ in the extended Zhang model is thus

$$
\begin{aligned}
G_{\mathrm{P}, \mathrm{N}, \text { fitting }}(T) & =G_{\mathrm{P}, \mathrm{N}, \mathrm{el}}(T)+G_{\mathrm{P}, \mathrm{N}, \text { in }}(T)+c_{0}, \\
G_{\mathrm{P}, \mathrm{N}, \mathrm{el}}(T) & =S_{\mathrm{e}}(T)\left\{1-\frac{P_{0}^{2}\left[1-\left(1-\eta_{\mathrm{P}} T^{3 / 2}\right)^{2}\right]}{1+P_{0}^{2}}\right\}, \quad \text { (7b) } \\
G_{\mathrm{P}, \mathrm{N}, \text { in }}(T) & =S_{\mathrm{e}}(T) a\left[\frac{1-P_{0}^{2}\left(1-\eta_{\mathrm{P}} T^{3 / 2}\right)^{2}}{1+P_{0}^{2}}\right] k_{\mathrm{B}} T \cdot f(T) .
\end{aligned}
$$

Figure 6 plots the fitting curves of Eq. (7) for the $G_{\mathrm{P}, \mathrm{N}}(T)$ shown in Fig. 4(a). The extended Zhang model reproduced the apparently complicated $G_{\mathrm{P}, \mathrm{N}}(T)$ for a wide $T$ range except $T<T_{1}$. The parameters giving the best fit, including $\eta_{\mathrm{P}}, a=$ $Q \cdot 2 S / E_{\mathrm{m}}$, and $E_{\mathrm{c}}^{\mathrm{P}}$, where $\eta_{\mathrm{P}}$ and $E_{\mathrm{c}}^{\mathrm{P}}$ are $\eta$ and $E_{\mathrm{c}}$ for the parallel alignment, and the resulting $f(290 \mathrm{~K})$ and $a \cdot f(290 \mathrm{~K})$ are summarized in Table II. The $\eta_{\mathrm{P}}$ value deduced from fitting, for example, for $\alpha=1.30 \mathrm{MTJ}$ was $3.29 \times 10^{-5} \mathrm{~K}^{-3 / 2} \pm 5 \%$. 


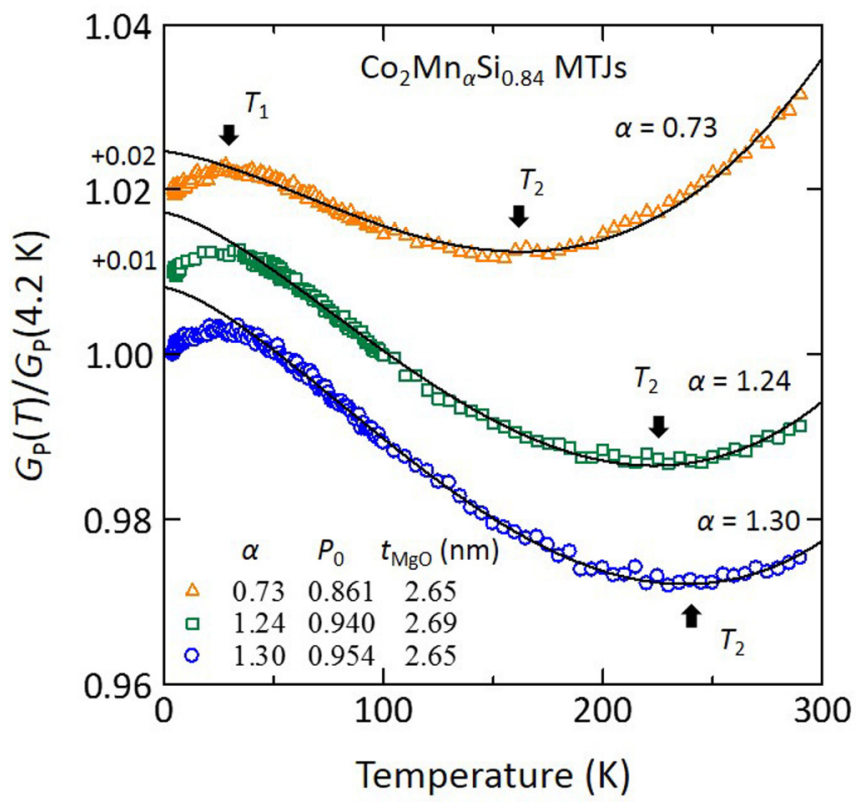

FIG. 6. Fitting curves of the extended Zhang model (solid lines) [Eq. (7)] for the experimental $G_{\mathrm{P}}(T)$ normalized by the respective values at $4.2 \mathrm{~K}$ of the CMS MTJs with $\alpha=0.73$ (open triangles), 1.24 (open squares), and 1.30 (open circles) in $\mathrm{Co}_{2} \mathrm{Mn}_{\alpha} \mathrm{Si}_{0.84}$ electrodes. The experimental data of $G_{\mathrm{P}}(T)$ are the same as shown in Fig. 4(a). The data of the normalized $G_{\mathrm{P}}(T)$ for $\alpha=0.73$ and 1.24 have respective offsets of +0.02 and +0.01 .

This relatively narrow error range was needed to reproduce the decreasing experimental $G_{\mathrm{P}, \mathrm{N}}(T)$ with increasing $T$ for $T_{1}<T<T_{2}$. The values of $a$ deduced for the CMS MTJs with various $\alpha$ values are almost equal. The value of $a$ in Eq. (7c) [and which is defined by Eq. (5c)] as calculated using the $T_{\mathrm{C}}$ of $985 \mathrm{~K}$ for CMS [32], a spin parameter $S$ from $1 / 2$ to $5 / 2$, and $\left|T^{\mathrm{d}}\right|^{2} /\left|T^{\mathrm{J}}\right|^{2}$ from 10 to 100 (according to Ref. [49]) ranged from 0.059 to $3.06 \mathrm{eV}^{-1}$. Thus, the deduced value of $3.01 \mathrm{eV}^{-1}$ for these MTJs was in a reasonable range. Here, $E_{\mathrm{c}}^{\mathrm{P}}$ slightly increased with $\alpha$ from $E_{\mathrm{c}}^{\mathrm{P}}=0.31 \mathrm{meV}$ for Mn-deficient $\alpha=0.73$ to $E_{\mathrm{c}}^{\mathrm{P}}=0.42 \mathrm{meV}$ for Mn-rich $\alpha=$ 1.30. Regarding $\eta_{\mathrm{P}}$, although the values deduced for the CMS MTJs with various $\alpha$ were within $\pm 5 \%$, they decreased with increasing $\alpha$ from $\eta_{\mathrm{P}}=3.70 \times 10^{-5} \mathrm{~K}^{-3 / 2}$ for $\alpha=0.73$ to $\eta_{\mathrm{P}}=3.29 \times 10^{-5} \mathrm{~K}^{-3 / 2}$ for $\alpha=1.30$, suggesting an increase in spin wave stiffness [68], or equivalently an increase in the energy of a spin wave with increasing $\alpha$. We also checked the validity of the deduced $\eta_{\mathrm{P}}$ values. To do so, we estimated the $P(290 \mathrm{~K})$ values from the respective $\eta_{P}$ values for various $\alpha$ by using Eq. (6). Here, we call the thus-estimated $P(290 \mathrm{~K})$ values $P^{\mathrm{TD}}(290 \mathrm{~K})$ and compare them with the corresponding $P(290 \mathrm{~K})$ values estimated from the respective TMR ratios at $290 \mathrm{~K}$ using the Julliere model of Eq. (1) $\left[P^{\mathrm{TMR}}(290 \mathrm{~K})\right]$. The $P^{\mathrm{TD}}(290 \mathrm{~K})$ values ranged from $P^{\mathrm{TD}}(290 \mathrm{~K})=0.704$ for $\alpha=0.73$ to $P^{\mathrm{TD}}(290 \mathrm{~K})=0.799$ for $\alpha=1.30$, which are comparable with the $P^{\mathrm{TMR}}(290 \mathrm{~K})$ values ranging from $P^{\mathrm{TMR}}(290 \mathrm{~K})=0.686$ for $\alpha=0.73$ to $P^{\mathrm{TMR}}(290 \mathrm{~K})=0.791$ for $\alpha=1.30$. This comparison suggests the deduced $\eta_{P}$ values are in a reasonable range even though the estimated $P^{\mathrm{TD}}(290 \mathrm{~K})$ values should not be regarded as rigorous.

Given the good agreement between the experimental and calculated $G_{\mathrm{P}, \mathrm{N}}(T)$ given by the extended Zhang model, we can now discuss the origin of the features in the experimental $G_{\mathrm{P}}(T)$ for the CMS MTJs with various $\alpha$ based on this model. The calculated $G_{\mathrm{P}, \mathrm{N}}(T)$ consists of basically two terms; one is the elastic tunneling term, and the other is the inelastic tunneling term. The variation with $T$ in the elastic tunneling term $\Delta G_{\mathrm{P}, \mathrm{N}, \mathrm{el}}(T)$ which is defined as $G_{\mathrm{P}, \mathrm{N}, \mathrm{el}}(T)-G_{\mathrm{P}, \mathrm{N}, \mathrm{el}}(0)$ is given as

$$
\Delta G_{\mathrm{P}, \mathrm{N}, \mathrm{el}}(T)=S_{\mathrm{e}}(T)\left\{1-\frac{P_{0}^{2}\left[1-\left(1-\eta_{\mathrm{P}} T^{3 / 2}\right)^{2}\right]}{1+P_{0}^{2}}\right\}-1,
$$

and the variation with $T$ in the inelastic tunneling term $\Delta G_{\mathrm{P}, \mathrm{N}, \text { in }}(T)$, which is identical to $G_{\mathrm{P}, \mathrm{N}, \text { in }}(T)$, is given by Eq. (7c). Figure 7(a) plots the variations from the respective values at $T=0 \mathrm{~K}$ in these two terms. Here, $G_{\mathrm{P}, \mathrm{N}, \mathrm{el}}(T)$ decreases $\left[\Delta G_{\mathrm{P}, \mathrm{N}, \mathrm{el}}(T)\right.$ is negative $]$ and $G_{\mathrm{P}, \mathrm{N}, \text { in }}(T)$ increases $\left[\Delta G_{\mathrm{P}, \mathrm{N}, \mathrm{in}}(T)\right.$ is positive] as $T$ increases. The latter feature is due to $f(T)>0$. Note that the former and latter features are those predicted by the original Shang and Zhang models, respectively. In the extended Zhang model, there is a competition between these two terms, resulting in a complicated $T$ dependence. Note that $\Delta G_{\mathrm{P}, \mathrm{N}, \mathrm{in}}(T)$ is smaller for higher $P_{0}$, which arises from the $P_{0}$ dependence of the factor $\left[1-P_{0}^{2}\left(1-\eta_{\mathrm{P}} T^{3 / 2}\right)^{2}\right] /\left(1+P_{0}^{2}\right)=C_{P}(T)$ in $\Delta G_{\mathrm{P}, \mathrm{N}, \text { in }}(T)$. The factor can be approximated as $C_{\mathrm{P}}(T) \approx$ $\left[1-P_{0}^{2}\left(1-2 \eta_{\mathrm{P}} T^{3 / 2}\right)\right] /\left(1+P_{0}^{2}\right)$, and the term of $2 \eta_{\mathrm{P}} T^{3 / 2}$ can be neglected when discussing the $\alpha$ dependence of $\Delta G_{\mathrm{P}, \mathrm{N}, \mathrm{in}}(T)$ because $2 \eta_{\mathrm{P}} T^{3 / 2}$ is much smaller than 1 even at $290 \mathrm{~K}$. Accordingly, we can replace the factor $C_{\mathrm{P}}(T)$ by a constant $\left(1-P_{0}^{2}\right) /\left(1+P_{0}^{2}\right)=\xi$, which decreases as $P_{0}$ increases and gets close to 0 as $P_{0}$ gets close to 1 . Thus, the positive $\Delta G_{\mathrm{P}, \mathrm{N}, \mathrm{in}}(T)$ is smaller for higher $P_{0}$ and gets close to 0 as $P_{0}$ gets close to 1 . This dependence originates from that $G_{\mathrm{P}, \text { in }}(T)$, which is due to spin-flip inelastic tunneling, is proportional to

TABLE II. Parameters of $\eta\left(\mathrm{K}^{-3 / 2}\right), a=Q \cdot 2 S / E_{\mathrm{m}}\left(\mathrm{eV}^{-1}\right), E_{\mathrm{c}}(\mathrm{meV})$, and $c_{0}$ for the CMS MTJs with various $\alpha$ shown in Table I deduced from fitting their respective normalized $G_{\mathrm{P}}(T)\left[=G_{\mathrm{P}}(T) / G_{\mathrm{P}}(4.2 \mathrm{~K})\right]$ by the proposed extended Zhang model, and the resulting $f(290 \mathrm{~K})$ [Eq. (3f)] and $a \cdot f(290 \mathrm{~K})$. The thus-obtained parameters $\eta$ and $E_{\mathrm{c}}$ for the parallel alignment are represented as $\eta_{\mathrm{P}}$ and $E_{\mathrm{c}}^{\mathrm{P}}$.

\begin{tabular}{|c|c|c|c|c|c|c|c|}
\hline$\alpha$ in $\mathrm{Co}_{2} \mathrm{Mn}_{\alpha} \mathrm{Si}_{0.84}$ & Fitting model & $\eta_{\mathrm{P}}\left(\mathrm{K}^{-3 / 2}\right)$ & $a=Q \cdot 2 S / E_{\mathrm{m}}\left(\mathrm{eV}^{-1}\right)$ & $E_{\mathrm{c}}^{\mathrm{P}}(\mathrm{meV})$ & $f(290 \mathrm{~K})$ & $a \cdot f(290 \mathrm{~K})$ & $c_{0}$ \\
\hline 0.73 & Extended Zhang model & $3.699 \times 10^{-5}$ & 3.004 & 0.311 & 4.392 & 13.2 & 0.005 \\
\hline 1.24 & Extended Zhang model & $3.319 \times 10^{-5}$ & 3.006 & 0.408 & 4.122 & 12.4 & 0.007 \\
\hline 1.30 & Extended Zhang model & $3.294 \times 10^{-5}$ & 3.008 & 0.415 & 4.105 & 12.3 & 0.008 \\
\hline
\end{tabular}



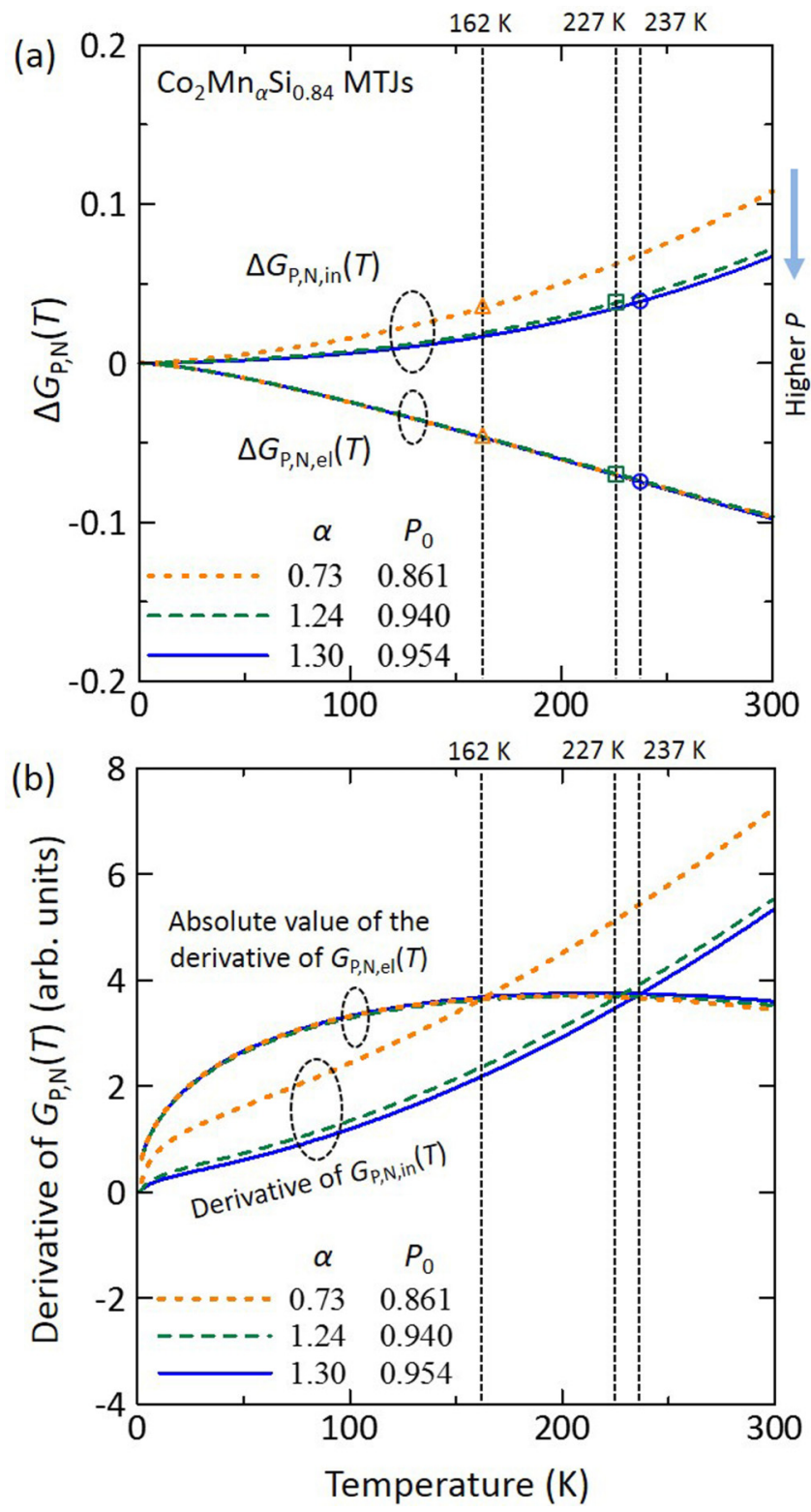

FIG. 7. (a) Variations with $T$ from the values at $T=0 \mathrm{~K}$ of the elastic tunneling term $\Delta G_{\mathrm{P}, \mathrm{N}, \mathrm{el}}(T)$ [Eq. (8)] and the inelastic tunneling term $\Delta G_{\mathrm{P}, \mathrm{N}, \text { in }}(T)$ [Eq. (7c)] obtained by fitting the experimental $G_{\mathrm{P}, \mathrm{N}}(T)$ by the extended Zhang model for CMS MTJs with $\alpha=0.73$ (dotted line), 1.24 (dashed line), and 1.30 (solid line). (b) Absolute values of the derivatives of $G_{\mathrm{P}, \mathrm{N}, \mathrm{el}}(T)$ and derivatives of $G_{\mathrm{P}, \mathrm{N}, \mathrm{in}}(T)$ for the CMS MTJs with $\alpha=0.73$ to 1.30 . The intersection of the $-d G_{\mathrm{P}, \mathrm{N}, \mathrm{el}}(T) / d T$ and $d G_{\mathrm{P}, \mathrm{N}, \text { in }}(T) / d T$ lines for each $\alpha$ in (b) determines $T_{2}$ to be $162 \mathrm{~K}$ for $\alpha=0.73,227 \mathrm{~K}$ for $\alpha=1.24$, and $237 \mathrm{~K}$ for $\alpha=1.30$. The triangles, squares, and circles shown in (a) are the $\Delta G_{\mathrm{P}, \mathrm{N}, \text { in }}\left(T_{2}\right)$ and $\Delta G_{\mathrm{P}, \mathrm{N}, \mathrm{el}}\left(T_{2}\right)$ values for the $\alpha=0.73,1.24$ and 1.30 MTJs, respectively.

the product of $2 \rho_{\mathrm{M}} \rho_{\mathrm{m}}$ ( $\rho_{\mathrm{m}}$ gets close to 0 for $P_{0}$ being close to 1) while the $G_{\mathrm{P}}$ at $T=0$ is proportional to the sum of $\rho_{\mathrm{M}}^{2}+\rho_{\mathrm{m}}^{2}$ $\left(\approx \rho_{\mathrm{M}}^{2}\right.$ for highly spin-polarized electrodes). On the other hand, the dominant term of $\Delta G_{\mathrm{P}, \mathrm{N}, \mathrm{el}}(T)$ [Eq. (8)] is approximated as $-2 P_{0}^{2} \eta_{\mathrm{P}} T^{3 / 2} /\left(1+P_{0}^{2}\right)$ because $\eta_{\mathrm{P}} T^{3 / 2} \ll 1$ and $S_{\mathrm{e}}(T)$ can be replaced with 1 when discussing the $\alpha$ dependence of $\Delta G_{\mathrm{P}, \mathrm{N}, \mathrm{el}}(T)$. Thus, the dependence of $\Delta G_{\mathrm{P}, \mathrm{N}, \mathrm{el}}(T)$ on $P_{0}$ is determined by the coefficient, $-2 P_{0}^{2} /\left(1+P_{0}^{2}\right)=B_{\mathrm{P}}$. Because $B_{\mathrm{P}}$ is negative, $G_{\mathrm{P}, \mathrm{N}, \mathrm{el}}(T)$ decrease as $T$ increases. Furthermore, $B_{\mathrm{P}}$ increases in magnitude as $P_{0}$ increases and gets close to -1 as $P_{0}$ gets close to 1 . This behavior of $B_{\mathrm{P}}$ is in contrast to that of the coefficient $\xi$, which gets close to 0 as $P_{0}$ gets close to 1 . Thus, the negative $\Delta G_{\mathrm{P}, \mathrm{N}, \mathrm{el}}(T)$ increases in magnitude as $P_{0}$ increases and gets close to a finite value as $P_{0}$ gets close to 1 . This $P_{0}$ dependence of $\Delta G_{\mathrm{P}, \mathrm{N}, \mathrm{el}}(T)$ is in contrast to that of the positive $\Delta G_{\mathrm{P}, \mathrm{N}, \mathrm{in}}(T)$ that gets close to 0 as $P_{0}$ gets close to 1 . Note the values of $\Delta G_{\mathrm{P}, \mathrm{N}, \mathrm{el}}(T)$ for $P_{0}$ ranging from 0.861 to 0.954 are almost equal. This is because (1) the magnitude in $d G_{\mathrm{P}, \mathrm{N}, \mathrm{el}}(T) / d P_{0}$ is smaller than that in $d G_{\mathrm{P}, \mathrm{N}, \mathrm{in}}(T) / d P_{0}$ (the former magnitude is half the latter), and (2) although its magnitude is smaller, a further increase in the magnitude of $d G_{\mathrm{P}, \mathrm{N}, \mathrm{el}}(T) / d P_{0}$ with increasing $P_{0}$ caused by the increase in $\alpha$ was mostly canceled by the smaller $\eta_{\mathrm{P}}$ for the larger $\alpha$. In summary, the negative $\Delta G_{\mathrm{P}, \mathrm{N}, \mathrm{el}}(T)$ becomes the more dominant factor in determining $G_{\mathrm{P}}(T)$ because $\Delta G_{\mathrm{P}, \mathrm{N}, \text { in }}(T)$ becomes smaller at higher $P_{0}$.

Next, let us discuss the origin of the $P_{0}$ dependence of $T_{2}$ shown in Fig. 5(a). The characteristic temperature $T_{2}$ corresponds to $d G_{\mathrm{P}}(T) / d T=0$. Figure $7(\mathrm{~b})$ plots the numerically obtained derivatives with respect to $T$ of the elastic tunneling terms, $G_{\mathrm{P}, \mathrm{N} \text {,el }}(T)$ [Eq. (7b)], and the inelastic term, $G_{\mathrm{P}, \mathrm{N}, \mathrm{in}}(T)$ [Eq. (7c)], for various $\alpha$ values, where $-d G_{\mathrm{P}, \mathrm{N}, \mathrm{el}}(T) / d T$ is plotted instead of $d G_{\mathrm{P}, \mathrm{N}, \mathrm{el}}(T) / d T$ because it is negative. The intersection of the $-d G_{\mathrm{P}, \mathrm{N}, \mathrm{el}}(T) / d T$ and $d G_{\mathrm{P}, \mathrm{N}, \text { in }}(T) / d T$ lines for each $\alpha$ determines $T_{2}$ to be $162 \mathrm{~K}$ for $P_{0}=0.861(\alpha=0.73)$ to $237 \mathrm{~K}$ for $P_{0}=0.954(\alpha=$ 1.30). The determined $T_{2}$ values agree with the corresponding experimental $T_{2}$ values because the experimental $T_{2}$ values were similarly determined from the $d G_{\mathrm{P}}(T) / d T=0$ point for the experimental $G_{\mathrm{P}}$. Because the derivative of $G_{\mathrm{P}, \mathrm{N}, \mathrm{in}}(T)$ decreased with increasing $P_{0}$, while $-d G_{\mathrm{P}, \mathrm{N} \text {,el }}(T) / d T$ remained almost constant for various $P_{0}$ values, the intersection point moved to a higher $T$ as $P_{0}$ increased from 0.861 to 0.954. The decrease in the magnitude of $d G_{\mathrm{P}, \mathrm{N}, \text { in }}(T) / d T$ with increasing $P_{0}$ originated from the coefficient $C_{\mathrm{P}}(T)=$ $\left[1-P_{0}^{2}\left(1-\eta_{\mathrm{P}} T^{3 / 2}\right)^{2}\right] /\left(1+P_{0}^{2}\right)$ in $G_{\mathrm{P}, \mathrm{N}, \text { in }}(T)$.

Now let us turn to the $P_{0}$ dependence of the maximum decrease in $G_{\mathrm{P}, \mathrm{N}}$ at $T_{2}$ [Fig. 5(b)]. Figure 7(a) plots straight lines corresponding to the respective $T_{2}$ values obtained from the intersection points in Fig. 7(b). The intersections of these straight lines with $\Delta G_{\mathrm{P}, \mathrm{N}, \mathrm{in}}(T)$ and $\Delta G_{\mathrm{P}, \mathrm{N}, \mathrm{el}}(T)$ for each $\alpha$ are their values at $T=T_{2}$. These plots show that $\Delta G_{\mathrm{P}, \mathrm{N}, \mathrm{el}}\left(T_{2}\right)$ increases in magnitude as $P_{0}$ increases from $P_{0}=0.861$ (for Mn-deficient $\alpha=0.73$ ) to $P_{0}=0.954$ (for Mn-rich $\alpha=$ 1.30), while $\Delta G_{\mathrm{P}, \mathrm{N}, \text { in }}\left(T_{2}\right)$ is almost constant for various $P_{0}$. The larger increase in the magnitude of $\Delta G_{\mathrm{P}, \mathrm{N}}\left(T_{2}\right)$ for higher $P_{0}$ is explained as follows. Here, $\Delta G_{\mathrm{P}, \mathrm{N}, \text { in }}(T)$ decreased in the whole $T$ range as $P_{0}$ increased. Thus, the contribution of $\Delta G_{\mathrm{P}, \mathrm{N}, \mathrm{in}}(T)$ basically decreased as $P_{0}$ increased. However, because each $\Delta G_{\mathrm{P}, \mathrm{N}, \mathrm{in}}(T)$ increased with increasing $T$, and $T_{2}$ increased as $P_{0}$ increased, $\Delta G_{\mathrm{P}, \mathrm{N}, \mathrm{in}}\left(T_{2}\right)$ was almost constant for various $P_{0}$. On the other hand, because (1) $\Delta G_{\mathrm{P}, \mathrm{N}, \mathrm{el}}(T)$ was almost independent of $P_{0}$ in the whole $T$ range, (2) the magnitude of $\Delta G_{\mathrm{P}, \mathrm{N}, \mathrm{el}}(T)$ increased with increasing $T$, and (3) $T_{2}$ increased as $P_{0}$ increased, the magnitude of $\Delta G_{\mathrm{P}, \mathrm{N}, \mathrm{el}}\left(T_{2}\right)$ 


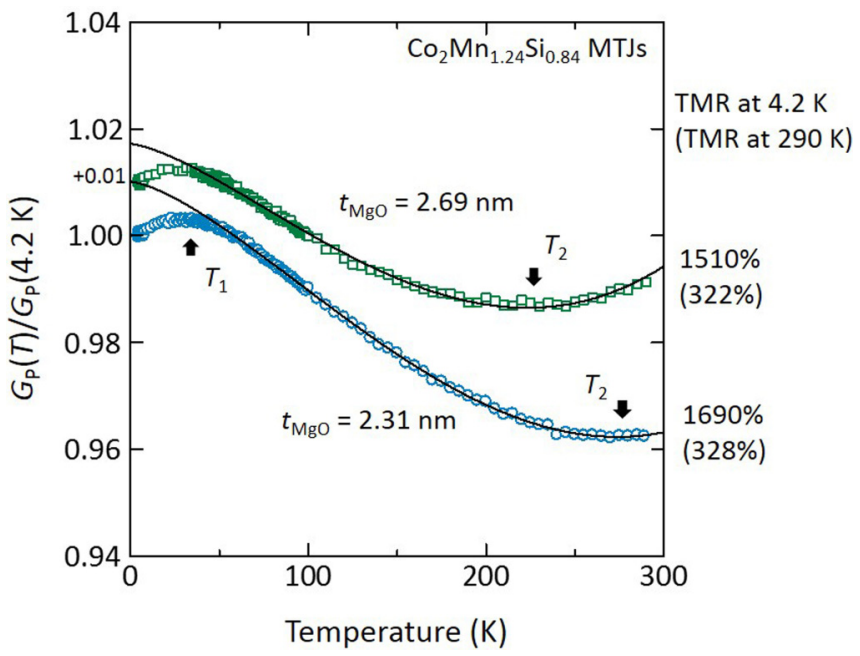

FIG. 8. Experimental $G_{\mathrm{P}}(T)$ normalized by its value at $4.2 \mathrm{~K}, G_{\mathrm{P}}(T) / G_{\mathrm{P}}(4.2 \mathrm{~K})$, of the $t_{\mathrm{MgO}}=2.31 \mathrm{~nm} \mathrm{CMS} \mathrm{MTJ}$ with $\mathrm{Co}_{2} \mathrm{Mn}_{1.24} \mathrm{Si}_{0.84}$ electrodes ( $\alpha=1.24$; open circles) along with that of the $t_{\mathrm{MgO}}=2.69 \mathrm{~nm}$ CMS MTJ ( $\alpha=1.24$; open squares) for comparison. These two MTJs were prepared with the same device fabrication process on the same epitaxial layer structure. The fitting curve of the extended Zhang model for the $t_{\mathrm{MgO}}=2.31 \mathrm{~nm} \mathrm{CMS} \mathrm{MTJ}$ is also shown along with that for the $t_{\mathrm{MgO}}=2.69 \mathrm{~nm} \mathrm{CMS} \mathrm{MTJ}$ for comparison. The experimental $G_{\mathrm{P}, \mathrm{N}}(T)$ data and fitting curve of the extended Zhang model for the $t_{\mathrm{MgO}}=2.69 \mathrm{~nm}$ MTJ $(\alpha=1.24)$ are the same as those shown in Fig. 6 . The data of the $G_{\mathrm{P}, \mathrm{N}}(T)$ for the $t_{\mathrm{MgO}}=2.69 \mathrm{~nm}$ MTJ have an offset of +0.01 .

increased with increasing $P_{0}$. Thus, the main factor that led to the $P_{0}$ dependence of the maximum decrease in $G_{\mathrm{P}, \mathrm{N}}$ at $T_{2}$ was $\Delta G_{\mathrm{P}, \mathrm{N}, \mathrm{in}}(T)$ decreasing with increasing $P_{0}$.

We will now discuss the influence of the smearing effect on the $T$ dependence of $G_{\mathrm{P}, \mathrm{N}}$ by analyzing two identical CMS MTJs with $\mathrm{Co}_{2} \mathrm{Mn}_{1.24} \mathrm{Si}_{0.84}$ electrodes prepared in the same device fabrication process on the same MTJ epitaxial layer structure but having different nominal $t_{\mathrm{MgO}}$ values of 2.31 and $2.69 \mathrm{~nm}$, where the $t_{\mathrm{MgO}}=2.69 \mathrm{~nm}$ MTJ $(\alpha=1.24)$ is the same as that analyzed above. Figure 8 compares the experimental $G_{\mathrm{P}, \mathrm{N}}(T)$ of the $t_{\mathrm{MgO}}=2.31 \mathrm{~nm} \operatorname{MTJ}(\alpha=1.24)$ with that of the $t_{\mathrm{MgO}}=2.69 \mathrm{~nm}$ MTJ $(\alpha=1.24)$ shown in Figs. 4(a) and 6. Here, the $t_{\mathrm{MgO}}=2.31 \mathrm{~nm}$ MTJ $(\alpha=1.24)$ showed a larger TMR ratio of $1690 \%$ at $4.2 \mathrm{~K}(328 \%$ at $290 \mathrm{~K})$ compared with $1510 \%$ at $4.2 \mathrm{~K}(322 \%$ at $290 \mathrm{~K})$ for the $t_{\mathrm{MgO}}=$ $2.69 \mathrm{~nm}$ MTJ $(\alpha=1.24)$, resulting in a larger $P(4.2 \mathrm{~K})$ of 0.946 for the $t_{\mathrm{MgO}}=2.31 \mathrm{~nm}$ MTJ $(\alpha=1.24)$ compared with the $P(4.2 \mathrm{~K})$ of 0.940 for the $t_{\mathrm{MgO}}=2.69 \mathrm{~nm} \mathrm{MTJ}(\alpha=1.24)$. Moreover, the experimental $G_{\mathrm{P}, \mathrm{N}}(T)$ of the $t_{\mathrm{MgO}}=2.31 \mathrm{~nm}$ MTJ $(\alpha=1.24)$ had a larger $T_{2}$ and a larger decrease at $T_{2}$. These features can be explained by the extended Zhang model, in particular by the two factors involved in the model: one is the larger $P_{0}$, leading to a further reduction in $G_{\mathrm{P}, \mathrm{N}, \text { in }}(T)$ compared with that for the $t_{\mathrm{MgO}}=2.69 \mathrm{~nm}$ MTJ $(\alpha=1.24)$; the other is a smaller $S_{\mathrm{e}}(T)$ value arising from the smaller $t_{\mathrm{MgO}}$, leading to a decrease in the unperturbed term with respect to the $T$-dependent $P$ in the elastic tunneling term, i.e., the first term of $S_{\mathrm{e}}(T) \cdot 1$ in Eq. (7b). Indeed, the extended Zhang model reproduced the experimental $G_{\mathrm{P}, \mathrm{N}}(T)$ of the $t_{\mathrm{MgO}}=2.31 \mathrm{~nm}$
$\operatorname{MTJ}(\alpha=1.24 ;$ Fig. 8$)$ as well as it did for the $t_{\mathrm{MgO}}=2.69 \mathrm{~nm}$ MTJ ( $\alpha=1.24$; Fig. 6). As listed in Table III, the deduced $\eta_{\mathrm{P}}$, $a$, and $E_{\mathrm{c}}^{\mathrm{P}}$ and resulting $a \cdot f(290 \mathrm{~K})$ for $t_{\mathrm{MgO}}=2.31 \mathrm{~nm}$ MTJ $(\alpha=1.24)$ are close to those deduced for the $t_{\mathrm{MgO}}=2.69 \mathrm{~nm}$ MTJ ( $\alpha=1.24$; Table II). This indicates that the different behaviors of these two MTJs can be reasonably explained by the $P_{0}$-dependent factor $\xi=\left(1-P_{0}^{2}\right) /\left(1+P_{0}^{2}\right)(\xi=0.056$ and 0.062 for the $t_{\mathrm{MgO}}=2.31$ and $2.69 \mathrm{~nm}$ MTJs, respectively) and $t_{\mathrm{MgO}}$-dependent factor $S_{\mathrm{e}}(T)$ in the extended Zhang model.

To reveal the problem associated with fitting $G_{\mathrm{P}}(T)$ by the Shang model with the smearing effect, we tentatively fitted the experimental $G_{\mathrm{P}, \mathrm{N}}(T)$ of the two MTJs analyzed above. We then compared the fitting results of the Shang model, in particular the deduced parameters, with those of the extended Zhang model. In the analysis of $G_{\mathrm{P}, \mathrm{N}}(T)$ of the Shang model, we fitted the experimental $G_{\mathrm{P}, \mathrm{N}}(T)$ by using three free parameters, i.e., $\eta$, the barrier height $\varphi$ in the smearing factor, and the additional small constant $c_{0}$. The fitting function for the normalized experimental $G_{\mathrm{P}, \mathrm{N}}(T)$ is

$$
G_{\mathrm{P}, \mathrm{N}, \mathrm{Shang}}(T)=S_{\mathrm{e}}(T)\left\{1-\frac{P_{0}^{2}\left[1-\left(1-\eta_{\mathrm{P}} T^{3 / 2}\right)^{2}\right]}{1+P_{0}^{2}}\right\}+c_{0} .
$$

The fitting curves for both MTJs (not shown) apparently reproduced the experimental $G_{\mathrm{P}, \mathrm{N}}(T)$ with the fitting parameters shown in Table III. On the other hand, the deduced barrier heights $\varphi$ in the factor $S_{\mathrm{e}}(T)$ of the Shang model, $\varphi_{\text {Shang }}$, for these MTJs were quite small (Table III) compared with the $\varphi=3.5 \mathrm{eV}$ assumed in the extended Zhang model. Furthermore, the $\varphi_{\text {Shang }}$ value of $1.08 \mathrm{eV}$ for the $t_{\mathrm{MgO}}=$ $2.31 \mathrm{~nm}$ MTJ was much smaller than the $\varphi_{\text {Shang }}$ value of $1.34 \mathrm{eV}$ of the $t_{\mathrm{MgO}}=2.69 \mathrm{~nm}$ MTJ that was needed to reproduce the $G_{\mathrm{P}, \mathrm{N}}(T)$ of the former MTJ featuring the larger $T_{2}$ and the larger decrease at $T_{2}$ in the experimental $G_{\mathrm{P}, \mathrm{N}}(T)$ than that of the latter MTJ. Such a significant difference in the deduced $\varphi$ values in the Shang model was caused because only the $S_{\mathrm{e}}(T)$ term is responsible for the increased component with increasing $T$ in the experimental $G_{\mathrm{P}, \mathrm{N}}(T)$ in the Shang model. However, such a significant difference is unreasonable because these MTJs were prepared from the same epitaxial layer structure. Furthermore, the linear relation in $\log R_{\mathrm{P}} A$ versus $t_{\mathrm{MgO}}$ for the CMS MTJs prepared on the epitaxial MTJ layer structure indicated that these MTJs are characterized by an identical barrier height. Thus, it is difficult to validate the fitting of the experimental $G_{\mathrm{P}, \mathrm{N}}(T)$ by the Shang model with the smearing effect even though the fitting apparently reproduced the experimental result.

Finally, we should note that the barrier height $\varphi$ was not definitely determined in this paper. However, its minimum value is estimated to be about $1.34 \mathrm{eV}$ or larger. This value is the same as the value deduced from fitting the $G_{\mathrm{P}, \mathrm{N}}(T)$ of the $t_{\mathrm{MgO}}=2.69 \mathrm{~nm}$ MTJ ( $\left.\alpha=1.24\right)$ by the Shang model with the smearing factor in which the contribution of inelastic tunneling is ignored, as described above. Even if we assume a wide $\varphi$ range of $3.5 \pm 1.5 \mathrm{eV}$, the factor $S_{\mathrm{e}}(290 \mathrm{~K})$ for a typical $t_{\mathrm{MgO}}$ value of $2.65 \mathrm{~nm}$ is weakly dependent on $\varphi$ in this range, and it only slightly changes from 1.10 for $\varphi=2.0 \mathrm{eV}$ to 1.04 for $\varphi=5.0 \mathrm{eV}$. Furthermore, $S_{\mathrm{e}}(290 \mathrm{~K})$ decreases with 
decreasing $t_{\mathrm{MgO}}$. Thus, although the smearing factor calculated with $\varphi=3.5 \mathrm{eV}$ corresponding to half the $\mathrm{MgO}$ energy gap has a certain margin of error, it would only slightly influence the results of the analysis in this paper, in particular by making a slight modification of the values of the deduced parameters.

In summary, our analysis of the experimental $G_{\mathrm{P}}(T)$ of the CMS MTJs characterized by the systematically varied $P(4.2 \mathrm{~K})$ showed that the extended Zhang model could consistently explain the complicated behavior of $G_{\mathrm{P}}(T)$. According to this model, $\Delta G_{\mathrm{P}, \mathrm{N}, \text { in }}(T)$ arising from spinflip inelastic tunneling via a magnon is much larger than $\Delta G_{\mathrm{P}, \mathrm{N}, \mathrm{el}}(T)$ arising from spin-conserving elastic tunneling in which $P$ decays with $T$ for MTJs characterized by a relatively low $P_{0}$. This results in an increase in $G_{\mathrm{P}}(T)$ with increasing $T$ from $4.2 \mathrm{~K}$ to room temperature. Thus, the analysis of the experimental $G_{\mathrm{P}}(T)$ of MTJs with a relatively low $P_{0}$ using the original Zhang model that ignores the decaying $P$ with $T$ could be a good approximation [51,52]. On the other hand, $\Delta G_{\mathrm{P}, \mathrm{N}, \text { in }}(T)$ decreases with increasing $P_{0}$ because of the decrease in the coefficient of $C_{\mathrm{P}}(T)$ or the effectively equivalent parameter $\xi=\left(1-P_{0}^{2}\right) /\left(1+P_{0}^{2}\right)$, resulting in a relative increase in $\Delta G_{\mathrm{P}, \mathrm{N}, \mathrm{el}}(T)$. This leads to competition between $\Delta G_{\mathrm{P}, \mathrm{N}, \mathrm{el}}(T)$ and $\Delta G_{\mathrm{P}, \mathrm{N}, \text { in }}(T)$. This competition caused the nonmonotonic $T$ dependence of $G_{\mathrm{P}}$ observed for the CMS MTJs. Thus, the proposed model provides a comprehensive understanding of the $T$ dependence of $G_{\mathrm{P}}$ of MTJs ranging from those characterized by a relatively low $P$ value to those characterized by a significantly high $P$ arising from half-metallicity.

\section{B. $T$ dependence of tunneling conductance for the antiparallel alignment}

Now let us analyze the $T$ dependence of $G_{\mathrm{AP}}$ of the CMS MTJs with various $\alpha$ values by using the extended Zhang model. For the antiparallel alignment, the smearing effect can be neglected for MTJs with high spin polarization. This is because the variation of $S_{\mathrm{e}}(T)$ from 4.2 to $290 \mathrm{~K}$ is about several percent which is much smaller than the relative variation in $G_{\mathrm{AP}}$ as $T$ increases from 4.2 to $290 \mathrm{~K}$. For the CMS MTJs, the normalized variation in $S_{\mathrm{e}}(T)$ at $290 \mathrm{~K}$, i.e., $\left[S_{\mathrm{e}}(290 \mathrm{~K})-S_{\mathrm{e}}(0 \mathrm{~K})\right] / S_{\mathrm{e}}(0 \mathrm{~K})$, is 0.056 for a $t_{\mathrm{MgO}}$ value of $2.65 \mathrm{~nm}$, as described above, while the normalized variation in $G_{\mathrm{AP}}(T)$ at $290 \mathrm{~K}$, i.e., $\left[G_{\mathrm{AP}}(290 \mathrm{~K})\right.$ $\left.-G_{\mathrm{AP}}(4.2 \mathrm{~K})\right] / G_{\mathrm{AP}}(4.2 \mathrm{~K})$, ranged from 1.33 for $\alpha=0.73$ to 3.60 for $\alpha=1.30$, as shown in Fig. 4(b). Thus, the smearing factor was ignored in the analysis of $G_{\mathrm{AP}}(T)$. Accordingly, the extended Zhang model for $G_{\mathrm{AP}}(T)$ is

$$
\begin{aligned}
G_{\mathrm{AP}}(T) & =G_{\mathrm{AP}, \mathrm{el}}(T)+G_{\mathrm{AP}, \text { in }}(T), \\
G_{\mathrm{AP}, \mathrm{el}}(T) & =Q_{1} \cdot 2 \rho_{\mathrm{M}}(T) \rho_{\mathrm{m}}(T), \\
G_{\mathrm{AP}, \text { in }}(T) & =Q_{2} k_{\mathrm{B}} T \cdot f(T) \cdot\left\{\left[\rho_{\mathrm{M}}(T)\right]^{2}+\left[\rho_{\mathrm{m}}(T)\right]^{2}\right\},
\end{aligned}
$$

where the coefficients $Q_{1}$ and $Q_{2}$ are given by Eqs. (3d) and (3e), respectively, the first term, $G_{\mathrm{AP}, \mathrm{el}}(T)$, of Eq. (10a) corresponds to spin-conserving elastic tunneling, and the second term, $G_{\mathrm{AP}, \text { in }}(T)$, corresponds to spin-flip inelastic tunneling. Here, $G_{\mathrm{AP}}$ at $T=0$ consists of spin-conserving elastic tunneling, which is expressed as

$$
G_{\mathrm{AP}}(T=0)=Q_{1} \cdot 2 \rho_{\mathrm{M}}(T=0) \rho_{\mathrm{m}}(T=0) .
$$

By assuming the $T$-dependent $P$ expressed by Eq. (6) with $\eta=\eta_{\mathrm{AP}}$ for the antiparallel alignment, $G_{\mathrm{AP}}(T)$ normalized by its value at $T=0, G_{\mathrm{AP}, \mathrm{N}}(T)$, is

$$
G_{\mathrm{AP}, \mathrm{N}}(T)=\frac{G_{\mathrm{AP}}(T)}{G_{\mathrm{AP}}(T=0)}=G_{\mathrm{AP}, \mathrm{N}, \mathrm{el}}(T)+G_{\mathrm{AP}, \mathrm{N}, \text { in }}(T),
$$

$$
\begin{aligned}
G_{\mathrm{AP}, \mathrm{N}, \mathrm{el}}(T) & =1+\frac{P_{0}^{2}\left[1-\left(1-\eta_{\mathrm{AP}} T^{3 / 2}\right)^{2}\right]}{1-P_{0}^{2}} \\
& =1+\Delta G_{\mathrm{AP}, \mathrm{N}, \mathrm{el}}(T), \\
G_{\mathrm{AP}, \mathrm{N}, \text { in }}(T) & =a\left[\frac{1+P_{0}^{2}\left(1-\eta_{\mathrm{AP}} T^{3 / 2}\right)^{2}}{1-P_{0}^{2}}\right] k_{\mathrm{B}} T \cdot f(T) \\
& =\Delta G_{\mathrm{AP}, \mathrm{N}, \mathrm{in}}(T),
\end{aligned}
$$

where the coefficient $a$ is given by Eq. (5c). We also included variations with $T$ in $G_{\mathrm{AP}, \mathrm{N}, \mathrm{el}}$ and $G_{\mathrm{AP}, \mathrm{N} \text {,in }}$ from the values at $T=0$, i.e., $\Delta G_{\mathrm{AP}, \mathrm{N}, \mathrm{el}}$ and $\Delta G_{\mathrm{AP}, \mathrm{N}, \text { in }}$, in Eqs. (12b) and (12c). If we assume a $T$-independent spin polarization, corresponding to $\eta_{\mathrm{AP}}=0$, Eq. (10) reduces to the original Zhang model,

\begin{tabular}{|c|c|c|c|c|c|c|c|c|c|}
\hline$t_{\mathrm{MgO}}(\mathrm{nm})$ & Fitting model & $\eta_{\mathrm{P}}\left(\mathrm{K}^{-3 / 2}\right)$ & $\varphi(\mathrm{eV})$ & $S_{\mathrm{e}}(290 \mathrm{~K})$ & $a=Q \cdot 2 S / E_{\mathrm{m}}\left(\mathrm{eV}^{-1}\right)$ & $E_{\mathrm{c}}^{\mathrm{AP}}(\mathrm{meV})$ & $f(290 \mathrm{~K})$ & $a \cdot f(290 \mathrm{~K})$ & $c_{0}$ \\
\hline \multirow[t]{2}{*}{2.31} & \multirow{2}{*}{$\begin{array}{l}\text { Extended Zhang model } \\
\text { Shang model with } \\
\text { smearing factor }\end{array}$} & $3.420 \times 10^{-5}$ & 3.5 & 1.042 & 3.009 & 0.486 & 3.951 & 11.9 & $\overline{0.010}$ \\
\hline & & $4.087 \times 10^{-5}$ & 1.08 & 1.147 & - & - & - & - & 0.012 \\
\hline \multirow[t]{2}{*}{2.69} & \multirow{2}{*}{$\begin{array}{l}\text { Extended Zhang model } \\
\text { Shang model with } \\
\text { smearing factor }\end{array}$} & $3.319 \times 10^{-5}$ & 3.5 & 1.058 & 3.006 & 0.408 & 4.122 & 12.4 & 0.007 \\
\hline & & $3.890 \times 10^{-5}$ & 1.34 & 1.162 & - & - & - & - & 0.009 \\
\hline
\end{tabular}
while if we ignore the second term of Eq. (10a), Eq. (10) reduces to the Shang model. Figure 9(a) plots the fitting curves

TABLE III. Parameters of $\eta_{\mathrm{P}}\left(\mathrm{K}^{-3 / 2}\right), a=Q \cdot 2 S / E_{\mathrm{m}}\left(\mathrm{eV}^{-1}\right), E_{\mathrm{c}}^{\mathrm{P}}(\mathrm{meV})$, and $c_{0}$ deduced from fitting $G_{\mathrm{P}, \mathrm{N}}(T)$ by the extended Zhang model, and the resulting $f(290 \mathrm{~K})$ and $a \cdot f(290 \mathrm{~K})$ for $\alpha=1.24 \mathrm{CMS}$ MTJ $\left(\mathrm{Co}_{2} \mathrm{Mn}_{1.24} \mathrm{Si}_{0.84}\right)$ with $t_{\mathrm{MgO}}$ of $2.31 \mathrm{~nm}$ are shown along with those values for $\alpha=1.24$ CMS MTJ with $t_{\mathrm{MgO}}$ of $2.69 \mathrm{~nm}$ (Table II) for comparison. The parameters of $\eta_{\mathrm{P}}\left(\mathrm{K}^{-3 / 2}\right)$, the barrier height $\varphi$, and the constant $c_{0}$ tentatively deduced from the fitting by the Shang model with the smearing factor for these two MTJs with $t_{\mathrm{MgO}}$ of 2.31 and $2.69 \mathrm{~nm}$ are also shown. The $t_{\mathrm{MgO}}=2.31 \mathrm{~nm} \mathrm{CMS} \mathrm{MTJ}(\alpha=1.24)$ had TMR ratios of $1690 \%$ at $4.2 \mathrm{~K}$ [corresponding to $P(4.2 \mathrm{~K})$ of 0.946 ] and $328 \%$ at $290 \mathrm{~K}$. 
TABLE IV. Parameters of $\eta\left(\mathrm{K}^{-3 / 2}\right), a=Q \cdot 2 S / E_{\mathrm{m}}\left(\mathrm{eV}^{-1}\right)$, and $E_{\mathrm{c}}(\mathrm{meV})$ for the $\mathrm{Co}_{2} \mathrm{Mn}_{1.30} \mathrm{Si}_{0.84}$ MTJ shown in Table I deduced from fitting $G_{\mathrm{AP}, \mathrm{N}}(T)$ by the Shang model, the extended Zhang model, and the original Zhang model. The thus-obtained parameters $\eta$ and $E_{\mathrm{c}}$ for the antiparallel alignment are represented as $\eta_{\mathrm{AP}}$ and $E_{\mathrm{c}}^{\mathrm{AP}}$.

\begin{tabular}{lcccccc}
\hline \hline$\alpha$ in $\mathrm{Co}_{2} \mathrm{Mn}_{\alpha} \mathrm{Si}_{0.84}$ & Fitting model & $\eta_{\mathrm{AP}}\left(\mathrm{K}^{-3 / 2}\right)$ & $a=Q \cdot 2 S / E_{\mathrm{m}}\left(\mathrm{eV}^{-1}\right)$ & $E_{\mathrm{c}}^{\mathrm{AP}}(\mathrm{meV})$ & $f(290 \mathrm{~K})$ & $a \cdot f(290 \mathrm{~K})$ \\
\hline 1.30 & Shang model & $4.231 \times 10^{-5}$ & - & - & - \\
& Extended Zhang model & $1.075 \times 10^{-5}$ & 1.032 & 0.178 & 4.947 \\
& Original Zhang model & - & 1.577 & 0.331 & 4.330 & 6.11 \\
\hline \hline
\end{tabular}

(a)
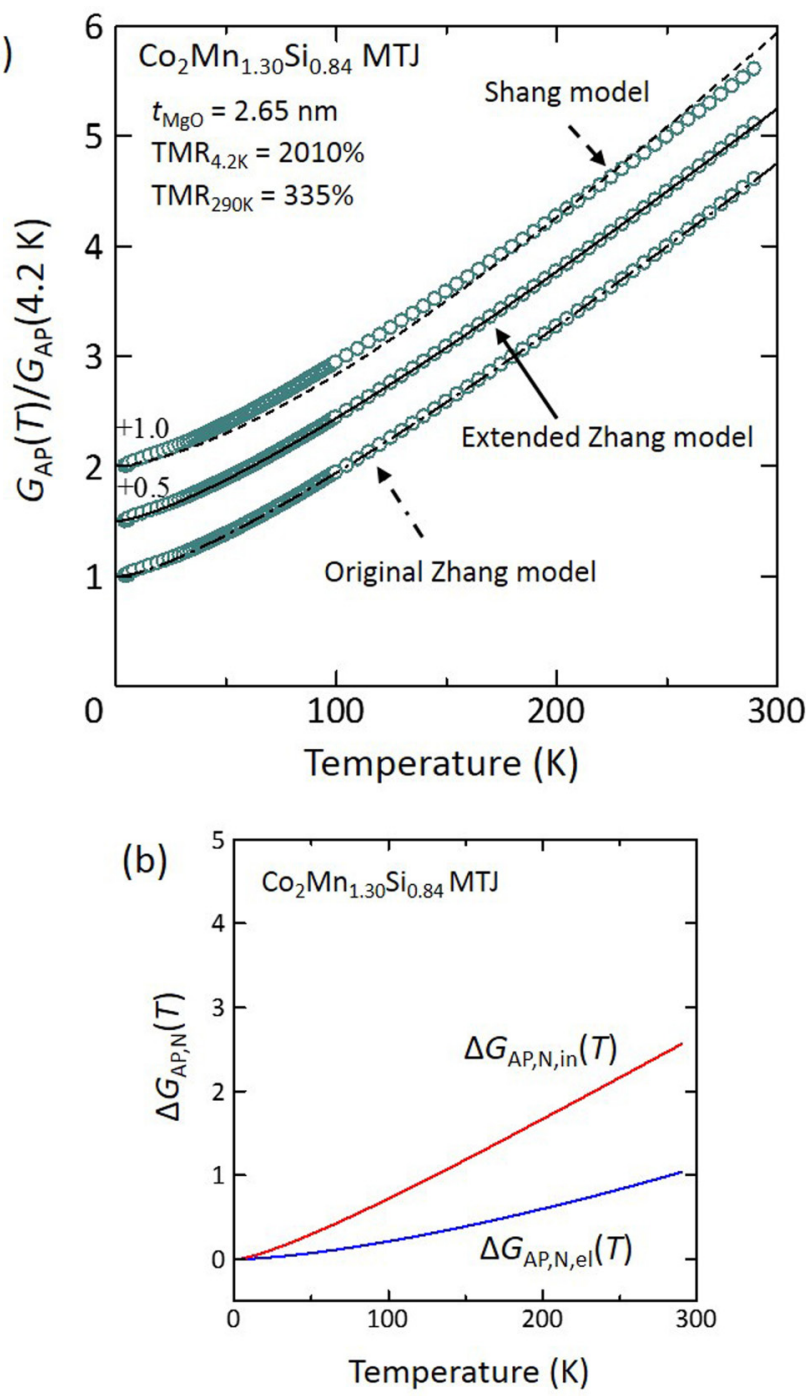

FIG. 9. (a) Comparison of fitting curves for $G_{\mathrm{AP}}(T)$ normalized by the value at $4.2 \mathrm{~K}$ of the CMS MTJ with $\mathrm{Co}_{2} \mathrm{Mn}_{1.30} \mathrm{Si}_{0.84}$ electrodes $(\alpha=1.30)$ by the original Zhang model (dashed-dotted line), the extended Zhang model (solid line), and the Shang model (dashed line). The experimental data (open circles) are the same as those of $\alpha=1.30$ shown in Figs. 3(a) and 4(b). The experimental $G_{\mathrm{AP}, \mathrm{N}}(T)$ data with the fitting curves of the extended Zhang and Shang models have respective offsets of +0.5 and +1.0 . (b) Decomposition of the variation of the experimental $G_{\mathrm{AP}, \mathrm{N}}(T)$ of $\alpha=1.30 \mathrm{CMS}$ MTJ into two components by fitting with the extended Zhang model: One is the inelastic tunneling term $\Delta G_{\mathrm{AP}, \mathrm{N}, \mathrm{in}}(T)$ [Eq. (12c)], and the other is the elastic tunneling term $\Delta G_{\mathrm{AP}, \mathrm{N}, \mathrm{el}}(T)$ [Eq. (12b)]. of the extended Zhang model of Eq. (12) for the experimental $G_{\mathrm{AP}, \mathrm{N}}(T)$ of the $\alpha=1.30 \mathrm{CMS}$ MTJ that had a giant TMR ratio of $2010 \%$ at $4.2 \mathrm{~K}$; the fitting curves of the original Zhang and Shang models are also plotted for comparison. The best fit parameters deduced from these fittings are listed in Table IV. It was found that all these fittings could reproduce the $G_{\mathrm{AP}, \mathrm{N}}(T)$. Among them, those of the original Zhang model and the extended Zhang model reproduced the experimental $G_{\mathrm{AP}, \mathrm{N}}(T)$ for a wide $T$ range from 4.2 to $290 \mathrm{~K}$, while the Shang model gave a fitting curve that showed slight but systematic deviations from the experimental $G_{\mathrm{AP}, \mathrm{N}}(T)$ for the entire $T$ range investigated. Furthermore, the Shang model's neglecting the contribution of spin-flip inelastic tunneling via a magnon could not be validated in light of our findings for the experimental $G_{\mathrm{P}, \mathrm{N}}(T)$ in Sec. III A. The essential point of the Shang model is that it explains the $T$ dependence of spin-dependent tunneling conductances, $G_{\mathrm{P}}$ and $G_{\mathrm{AP}}$, by only decreasing the spin polarization with increasing $T$ within a framework of spin-conserving elastic tunneling. Thus, the dynamic effect, i.e., the spin-flip inelastic tunneling via a magnon, at finite temperatures is effectively incorporated into the $T$ dependence of the spin polarization, meaning that the Shang model can roughly reproduce the experimental $G_{\mathrm{AP}, \mathrm{N}}(T)$ but with slight and systematic deviations. Similarly, the original Zhang model's neglecting the effect of the $T$ dependence of $P$ on elastic tunneling could not be validated even though it could reproduce the experimental $G_{\mathrm{AP}, \mathrm{N}}(T)$ as well as the extended Zhang model could do. Regarding the validity of the deduced parameters shown in Table IV, both coefficients $a$ deduced by the extended and original Zhang models are in the expected range, as described in Sec. III A.

Figure 9(b) decomposes the variation with $T$ in the experimental $G_{\mathrm{AP}}$ of $\alpha=1.30 \mathrm{MTJ}$ normalized by its value at $4.2 \mathrm{~K}$, i.e., $\Delta G_{\mathrm{AP}, \mathrm{N}}(T)=G_{\mathrm{AP}}(T) / G_{\mathrm{AP}}(4.2 \mathrm{~K})-1$, into a component arising from the elastic tunneling term $\Delta G_{\mathrm{AP}, \mathrm{N}, \mathrm{el}}(T)$ [Eq. (12b)] and a component arising from the inelastic tunneling term $\Delta G_{\mathrm{AP}, \mathrm{N}, \text { in }}(T)$ [Eq. (12c)]. Although $\Delta G_{\mathrm{AP}, \mathrm{N}, \text { in }}(T)$ was larger than $\Delta G_{\mathrm{AP}, \mathrm{N}, \mathrm{el}}(T)$, these two contributions were more or less comparable. This result of the analysis of the extended Zhang model for $G_{\mathrm{AP}, \mathrm{N}}(T)$ clearly indicates that the decrease in the TMR ratio at $290 \mathrm{~K}$ can be attributed to a static effect, i.e., spin-conserving elastic tunneling with decaying $P$ with $T$, and a dynamic effect, i.e., spin-flip inelastic tunneling, as described above. This result also suggests the actual $P$ value at $290 \mathrm{~K}$ would be higher than the $P^{\mathrm{TMR}}(290 \mathrm{~K})$ value. Indeed, the $P^{\mathrm{TD}}(290 \mathrm{~K})$ value for the $\alpha=1.30 \mathrm{MTJ}$ as calculated from the $\eta_{\mathrm{AP}}$ value by the fitting of the extended Zhang model (Table IV) was 0.903, which was obviously higher than the $P^{\text {TMR }}(290 \mathrm{~K})$ of 0.791 . Note that the tentatively calculated 


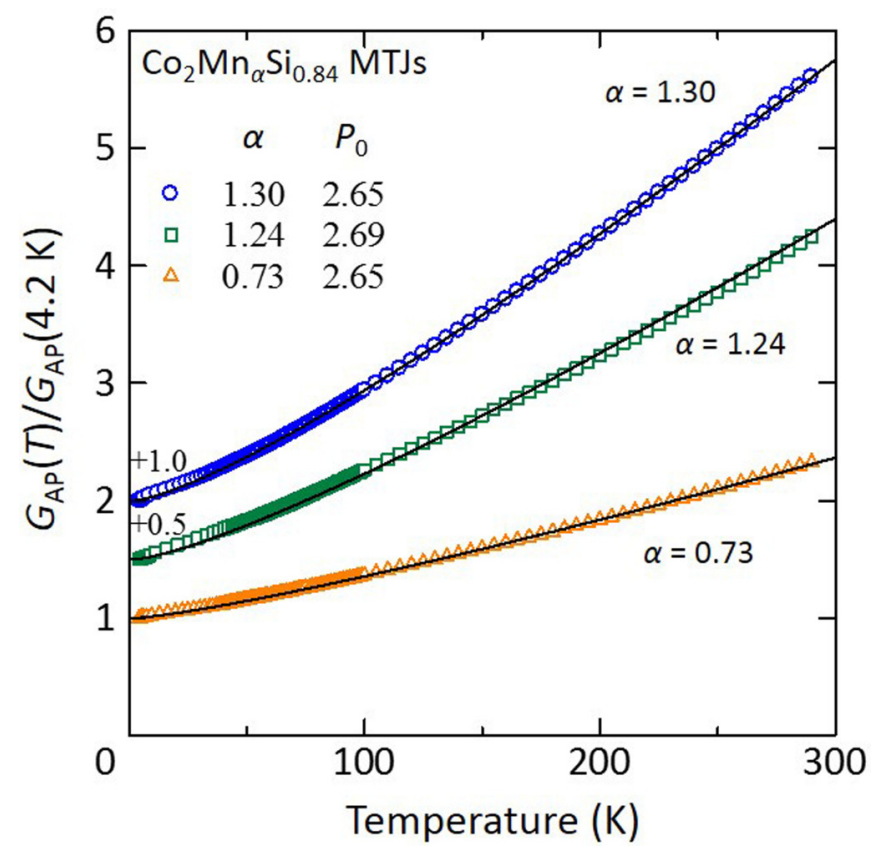

FIG. 10. Fitting curves (solid lines) for the experimental $G_{\mathrm{AP}}(T)$ normalized by the respective values at $4.2 \mathrm{~K}$ by the extended Zhang model [Eq. (12)] for the CMS MTJs with various $\alpha$ in $\mathrm{Co}_{2} \mathrm{Mn}_{\alpha} \mathrm{Si}_{0.84}$ electrodes. The experimental $G_{\mathrm{AP}, \mathrm{N}}(T)$ are the same as shown in Fig. 4(b) and are shown as open triangles, open squares, and open circles for $\alpha=0.73,1.24$, and 1.30 , respectively. The normalized $G_{\mathrm{AP}}(T)$ for $\alpha=1.24$ and 1.30 have respective offsets of +0.5 and +1.0 .

$P^{\mathrm{TD}}(290 \mathrm{~K})$ value from the $\eta_{\mathrm{AP}}$ value by the fitting of the Shang model was 0.755 , which was comparable to the $P^{\mathrm{TMR}}(290 \mathrm{~K})$. The lower $P^{\mathrm{TD}}(290 \mathrm{~K})$ value by the Shang model than that by the extended Zhang model was reasonable because the Shang model ignored the contribution of inelastic tunneling. Note also that the $P^{\mathrm{TD}}(290 \mathrm{~K})$ of 0.799 as calculated from $\eta_{\mathrm{P}}$ of the extended Zhang model in Sec. III A was much smaller than that as calculated from $\eta_{\mathrm{AP}}$ of the extended Zhang model. This difference in the absolute values of $\eta_{\mathrm{P}}$ and $\eta_{\mathrm{AP}}$ is ascribed to the simple relation of Eq. (6) phenomenologically describing the $T$ dependence of $P$ by a parameter $\eta$. Taking into account, however, that the dominant factor that decreases the TMR ratio at $290 \mathrm{~K}$ is the $T$ dependence of $G_{\mathrm{AP}}(T)$, the semiquantitative comparison of the $P^{\mathrm{TD}}(290 \mathrm{~K})$ values estimated from the $\eta_{\mathrm{AP}}$ values (rather than those from the $\eta_{\mathrm{P}}$ values) is more probable.

Figure 10 plots the fitting curves of the extended Zhang model [Eq. (12)] for the experimental $G_{\mathrm{AP}, \mathrm{N}}(T)$ for $\alpha$ ranging from 0.73 to 1.30 . The extended $Z$ hang model reproduced the
$G_{\mathrm{AP}, \mathrm{N}}(T)$ for all of the CMS MTJs over a wide $T$ range from 4.2 to $290 \mathrm{~K}$. The best fit parameters and resulting $f(290 \mathrm{~K})$ and $a \cdot f(290 \mathrm{~K})$ are summarized in Table $\mathrm{V}$. The quantities $\gamma_{\mathrm{AP}}=G_{\mathrm{AP}}(290 \mathrm{~K}) / G_{\mathrm{AP}}(4.2 \mathrm{~K})$, which represent the degree of the $T$ dependence of $G_{\mathrm{AP}}$, are also shown in Table V. The coefficients $a$ for these MTJs were almost identical. Here, $E_{\mathrm{c}}$ for the antiparallel alignment also increased from $0.063 \mathrm{meV}$ for Mn-deficient $\alpha=0.73$ to $0.18 \mathrm{meV}$ for Mn-rich $\alpha=1.30$ as $E_{\mathrm{c}}$ for the parallel alignment increased. This dependence led to a slight decrease in the product $a \cdot f(290 \mathrm{~K})$ as $\alpha$ increased from Mn-deficient to Mn-rich. Now let us turn to the origin of the stronger $T$ dependence of $G_{\mathrm{AP}, \mathrm{N}}(T)$ for higher $P(4.2 \mathrm{~K})$ [Fig. 4(b)], resulting in the larger $\gamma_{\mathrm{AP}}$ for higher $P(4.2 \mathrm{~K})$. Note that the factor, $\left[1+P_{0}^{2}\left(1-\eta_{\mathrm{AP}} T^{3 / 2}\right)^{2}\right] /\left(1-P_{0}^{2}\right)=C_{\mathrm{AP}}(T)$, in $\Delta G_{\mathrm{AP}, \mathrm{N}, \text { in }}(T)$ [Eq. (12c)] can be approximated as $C_{\mathrm{AP}}(T) \approx$ $\left[1+P_{0}^{2}\left(1-2 \eta_{\mathrm{AP}} T^{3 / 2}\right)\right] /\left(1-P_{0}^{2}\right)$, and the term $2 \eta_{\mathrm{AP}} T^{3 / 2}$ can be neglected in the discussion on the $\alpha$ dependence of $\gamma_{\mathrm{AP}}$ because $2 \eta_{\mathrm{AP}} T^{3 / 2}$ even at $290 \mathrm{~K}$ was much smaller than 1 . Accordingly, we can replace the factor $C_{\mathrm{AP}}(\mathrm{T})$ by a constant $\left(1+P_{0}^{2}\right) /\left(1-P_{0}^{2}\right)=1 / \xi$, which is just the inverse of the coefficient appearing in $\Delta G_{\mathrm{P}, \mathrm{N}, \text { in }}(T)$ for $2 \eta_{\mathrm{P}} T^{3 / 2} \ll 1.1 / \xi$ increases with $P_{0}$ and its values for the MTJs with various $\alpha$ are shown in Table $\mathrm{V}$. This dependence originates from the fact that the inelastic tunneling term in $G_{\mathrm{AP}}$ at finite temperatures, $G_{\mathrm{AP}, \text { in }}(T)$, is proportional to the sum of $\rho_{\mathrm{M}}^{2}+\rho_{\mathrm{m}}^{2}$, while $G_{\mathrm{AP}}$ at $T=0 \mathrm{~K}$ is proportional to the product $2 \rho_{\mathrm{M}} \rho_{\mathrm{m}}$. Furthermore, the term $P_{0}^{2}\left[1-\left(1-\eta_{\mathrm{AP}} T^{3 / 2}\right)^{2}\right] /\left(1-P_{0}^{2}\right)$ in $\Delta G_{\mathrm{AP}, \mathrm{N}, \mathrm{el}}(T)$ [Eq. (12b)] can be approximated as $2 P_{0}^{2} \eta_{\mathrm{AP}} T^{3 / 2} /\left(1-P_{0}^{2}\right)$ because $\eta_{\mathrm{AP}} T^{3 / 2} \ll 1$. The coefficient $2 P_{0}^{2} /\left(1-P_{0}^{2}\right)=B_{\mathrm{AP}}$ is also an increasing function of $P_{0}$. Note that the coefficient of $B_{\mathrm{AP}}$ in $\Delta G_{\mathrm{AP}, \mathrm{N}, \mathrm{el}}(T)$ is close to the coefficient $1 / \xi$ in $\Delta G_{\mathrm{AP}, \mathrm{N}, \text { in }}(T)$ for high $P_{0}$. We normalized $B_{\mathrm{AP}}, 1 / \xi$, and $a \cdot f(290 \mathrm{~K})$ for various $\alpha$ by their values at $\alpha=0.73$ and plotted them as a function of $\alpha$ in Fig. 11(a), which clearly shows significant increases in the normalized $1 / \xi$ and $B_{\mathrm{AP}}$ as $\alpha$ increases from $\mathrm{Mn}$-deficient to $\mathrm{Mn}$-rich. This dependence originated from the increase in $P_{0}$, while the product $a$. $f(290 \mathrm{~K})$ was almost independent of $\alpha$ even though it slightly decreased with increasing $\alpha$. Thus, it was revealed that the dominant factors determining the $\alpha$ dependence of $\gamma_{\mathrm{AP}}$ were the coefficient $1 / \xi$ in $\Delta G_{\mathrm{AP}, \mathrm{N}, \text { in }}(T)$ and the almost equivalent coefficient $B_{\mathrm{AP}}$ in $\Delta G_{\mathrm{AP}, \mathrm{N}, \mathrm{el}}(T)$. Because of the similar values of $1 / \xi$ and $B_{\mathrm{AP}}$ for high $P_{0}$, the dominant factor was $1 / \xi$ (or equivalently $B_{\mathrm{AP}}$ ). Figure $11(\mathrm{~b})$ plots how $\gamma_{\mathrm{AP}}$ depends on $1 / \xi$ for the CMS MTJs with $\mathrm{Co}_{2} \mathrm{Mn}_{\alpha} \mathrm{Si}_{0.84}$ electrodes of this paper as well as for the CMS MTJs with $\mathrm{Co}_{2} \mathrm{Mn}_{\alpha} \mathrm{Si}_{0.96}$ electrodes reported in Ref. [12], where the $G_{\mathrm{AP}, \mathrm{N}}(T)$ data were analyzed using the original Zhang model. We reexamined the $G_{\mathrm{P}, \mathrm{N}}(T)$ and $G_{\mathrm{AP}, \mathrm{N}}(T)$ data of the $\mathrm{Co}_{2} \mathrm{Mn}_{\alpha} \mathrm{Si}_{0.96}$ MTJs by using the

TABLE V. Parameters of $\eta_{\mathrm{AP}}\left(\mathrm{K}^{-3 / 2}\right), a=Q \cdot 2 S / E_{\mathrm{m}}\left(\mathrm{eV}^{-1}\right)$, and $E_{\mathrm{c}}^{\mathrm{AP}}(\mathrm{meV})$ for the CMS MTJs with various $\alpha$ shown in Table I deduced from fitting their respective $G_{\mathrm{AP}, \mathrm{N}}(T)$ by the extended Zhang model, and the resulting $f(290 \mathrm{~K})$ and $a \cdot f(290 \mathrm{~K})$. The quantities of $\gamma_{\mathrm{AP}}=G_{\mathrm{AP}}(290 \mathrm{~K}) / G_{\mathrm{AP}}(4.2 \mathrm{~K})$ and $1 / \xi$ defined by $1 / \xi=\left(1+P_{0}^{2}\right) /\left(1-P_{0}^{2}\right)$ are also shown.

\begin{tabular}{llccrrrr}
\hline \hline$\alpha$ in $\mathrm{Co}_{2} \mathrm{Mn}_{\alpha} \mathrm{Si}_{0.84}$ & $\eta_{\mathrm{AP}}\left(\mathrm{K}^{-3 / 2}\right)$ & $a=Q \cdot 2 S / E_{\mathrm{m}}\left(\mathrm{eV}^{-1}\right)$ & $E_{\mathrm{c}}^{\mathrm{AP}}(\mathrm{meV})$ & $f(290 \mathrm{~K})$ & $a \cdot f(290 \mathrm{~K})$ & $\gamma_{\mathrm{AP}}$ & $1 / \xi$ \\
\hline 0.73 & $1.227 \times 10^{-5}$ & 1.014 & 0.063 & 5.984 & 6.07 & 2.33 \\
1.24 & $1.113 \times 10^{-5}$ & 1.004 & 0.150 & 5.118 & 5.14 & 3.75 \\
1.30 & $1.075 \times 10^{-5}$ & 1.032 & 0.178 & 4.947 & 5.11 & 4.60 & 21.1 \\
\hline \hline
\end{tabular}



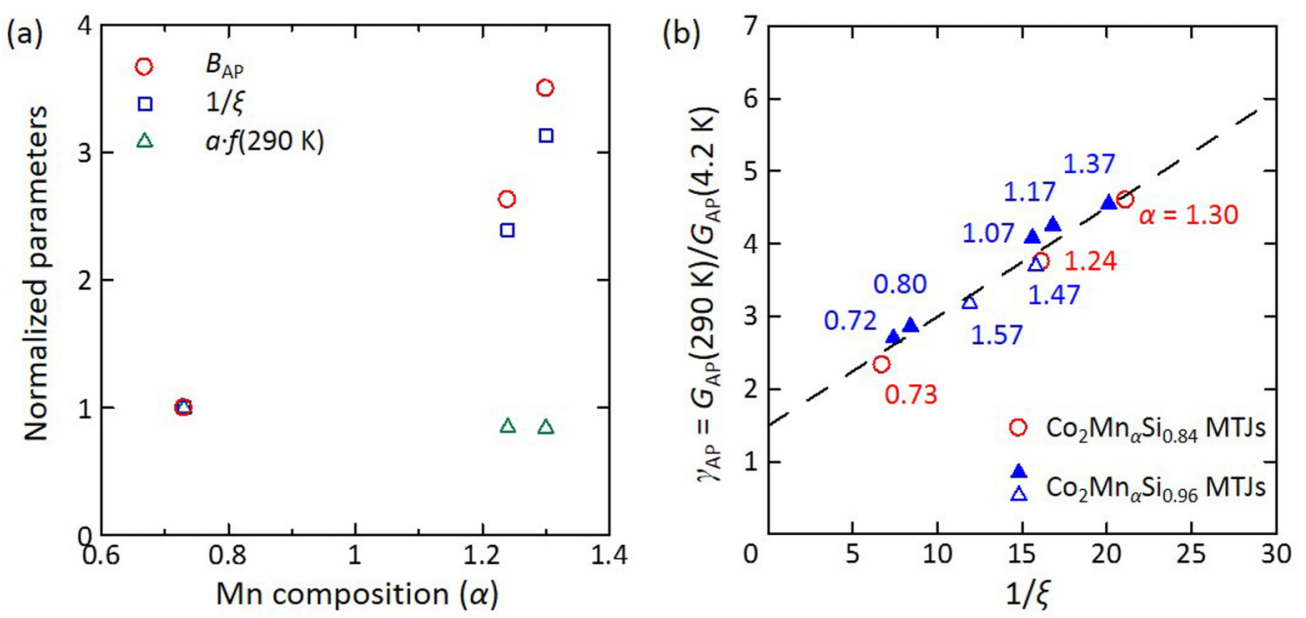

FIG. 11. (a) Dependence of the coefficient $B_{\mathrm{AP}}=2 P_{0}^{2} /\left(1-P_{0}^{2}\right)$, the parameter $1 / \xi=\left(1+P_{0}^{2}\right) /\left(1-P_{0}^{2}\right)$, and the product $a \cdot f(290 \mathrm{~K})$ on Mn composition $\alpha$ in $\mathrm{Co}_{2} \mathrm{Mn}_{\alpha} \mathrm{Si}_{0.84}$ electrodes, where these values are normalized by the respective values for $\alpha=0.73$. (b) Dependence of the parameter $\gamma_{\mathrm{AP}}=G_{\mathrm{AP}}(290 \mathrm{~K}) / G_{\mathrm{AP}}(4.2 \mathrm{~K})$ on $1 / \xi$ for CMS MTJs with $\mathrm{Co}_{2} \mathrm{Mn}_{\alpha} \mathrm{Si}_{0.84}$ electrodes (open circles). Dependence of $\gamma_{\mathrm{AP}}$ on $1 / \xi$ for CMS MTJs with $\mathrm{Co}_{2} \mathrm{Mn}_{\alpha} \mathrm{Si}_{0.96}$ electrodes reported in Ref. [12] are also plotted (solid and open triangles), where solid triangles are for $\alpha$ being smaller than a certain critical $\alpha$ value, $\alpha_{\mathrm{c}}$, for $\mathrm{Co}_{2} \mathrm{Mn}_{\alpha} \mathrm{Si}_{0.96}$ electrodes, and open triangles are for $\alpha$ being larger than $\alpha_{\mathrm{c}}$ [12].

extended Zhang model and found that the data could also be consistently explained by it. The almost linear dependence of $\gamma_{\mathrm{AP}}$ on $1 / \xi$ for the two series of CMS MTJs also clearly shows the dominant factor for the larger $\gamma_{\mathrm{AP}}$ for higher $P(4.2 \mathrm{~K})$ was the coefficient $1 / \xi$ which increased with increasing $P(4.2 \mathrm{~K})$. This result also indicates that the larger $\gamma_{\mathrm{AP}}$ for higher $P(4.2 \mathrm{~K})$ is the intrinsic spin-dependent tunneling property of MTJs [12], which was explained by the extended Zhang model.

In summary, the experimental $G_{\mathrm{AP}}(T)$, including its stronger $T$ dependence for higher $P$ at $4.2 \mathrm{~K}$, was consistently explained with the extended Zhang model. Our findings that both spin-flip inelastic tunneling via a thermally excited magnon and spin-conserving elastic tunneling in which $P$ decays with increasing $T$ play key roles in determining the $T$ dependence of $G_{\mathrm{P}}$ and $G_{\mathrm{AP}}$ are applicable to MTJs with a wide range of $P_{0}$. Moreover, our findings provide a unified picture of the origin of the $T$ dependence of the spin-dependent tunneling conductance of MTJs, including those with highly spin-polarized electrodes.

\section{CONCLUSION}

We investigated the key tunneling mechanisms that determine the temperature $(T)$ dependence of the spindependent tunneling conductances, $G_{\mathrm{P}}$ and $G_{\mathrm{AP}}$, of MTJs. To do so, we measured the $G_{\mathrm{P}}(T)$ and $G_{\mathrm{AP}}(T)$ of highquality $\mathrm{CMS} / \mathrm{MgO} / \mathrm{CMS}$ MTJs featuring systematically varied tunneling spin polarizations at $4.2 \mathrm{~K}[P(4.2 \mathrm{~K})](\alpha$ in the $\mathrm{Co}_{2} \mathrm{Mn}_{\alpha} \mathrm{Si}_{0.84}$ electrodes was varied) that exhibited giant TMR ratios. Although the $T$ dependence of the normalized $G_{\mathrm{P}}\left[G_{\mathrm{P}, \mathrm{N}}(T)=G_{\mathrm{P}}(T) / G_{\mathrm{P}}(4.2 \mathrm{~K})\right]$ was significantly weak compared with that of the normalized
$G_{\mathrm{AP}}\left[=G_{\mathrm{AP}}(T) / G_{\mathrm{AP}}(4.2 \mathrm{~K})\right], G_{\mathrm{P}, \mathrm{N}}$ showed a notable $T$ dependence in which it decreased with increasing $T$ from $T_{1}$ of about $30 \mathrm{~K}$ to $T_{2}$ ranging from about 162 to $237 \mathrm{~K}$, wherein $T_{2}$ depended on $\alpha$; then it increased for $T>T_{2}$. Furthermore, $T_{2}$ increased, and the maximum decrease in $G_{\mathrm{P}, \mathrm{N}}$ at $T_{2}$ increased as $P(4.2 \mathrm{~K})$ increased. These features indicated that an analysis of the experimental $G_{\mathrm{P}, \mathrm{N}}(T)$ is critical to elucidating the origin of the $T$ dependence of the spindependent tunneling conductance. To explain these features of $G_{\mathrm{P}, \mathrm{N}}(T)$, we developed an extension of the Zhang model for $G_{\mathrm{P}}(T)$ and $G_{\mathrm{AP}}(T)$ that took into account both spin-conserving elastic tunneling wherein $P$ decreases with increasing $T$, and in turn decreases $G_{\mathrm{P}}$ with $T$, and spin-flip inelastic tunneling via a thermally excited magnon that increases $G_{\mathrm{P}}$ with $T$. Accordingly, the complicated nonmonotonic behavior of the experimental $G_{\mathrm{P}, \mathrm{N}}(T)$ could be fitted with reasonable values of the parameters of the proposed model. The observed $P(4.2 \mathrm{~K})$ dependence of $G_{\mathrm{P}, \mathrm{N}}(T)$ was also consistently explained by the proposed model through a decrease in the contribution of spin-flip inelastic tunneling with increasing $P(4.2 \mathrm{~K})$. The normalized $G_{\mathrm{AP}}(T)$, including its stronger $T$ dependence for higher $P(4.2 \mathrm{~K})$, was also consistently explained. The proposed model provides a comprehensive understanding of $G_{\mathrm{P}}(T)$ and $G_{\mathrm{AP}}(T)$ of not only half-metallic MTJs but also MTJs with a wide range of $P$ at $0 \mathrm{~K}$.

\section{ACKNOWLEDGMENTS}

This paper was partly supported by Grants-in-Aid for Scientific Research (Grants No. 25286039 and No. 15K13960) from the Japan Society for the Promotion of Science (JSPS). B.H. was also supported by a scholarship from the China Scholarship Council (File No. 201306460029). 
[1] S. A. Wolf, D. D. Awschalom, R. A. Buhrman, J. M. Daughton, S. von Molnár, M. L. Roukes, A. Y. Chtchelkanova, and D. M. Treger, Science 294, 1488 (2001).

[2] I. Žutić, J. Fabian, and S. Das Sarma, Rev. Mod. Phys. 76, 323 (2004).

[3] R. A. de Groot, F. M. Mueller, P. G. van Engen, and K. H. J. Buschow, Phys. Rev. Lett. 50, 2024 (1983).

[4] C. Felser, G. H. Fecher, and B. Balke, Angew. Chem., Int. Ed. 46, 668 (2007).

[5] K. Inomata, S. Okamura, R. Goto, and N. Tezuka, Jpn. J. Appl. Phys. 42, L419 (2003).

[6] S. Kämmerer, A. Thomas, A. Hütten, and G. Reiss, Appl. Phys. Lett. 85, 79 (2004).

[7] Y. Sakuraba, M. Hattori, M. Oogane, Y. Ando, H. Kato, A. Sakuma, T. Miyazaki, and H. Kubota, Appl. Phys. Lett. 88, 192508 (2006).

[8] T. Ishikawa, T. Marukame, H. Kijima, K.-i. Matsuda, T. Uemura, M. Arita, and M. Yamamoto, Appl. Phys. Lett. 89, 192505 (2006).

[9] T. Ishikawa, H.-x. Liu, T. Taira, K.-i. Matsuda, T. Uemura, and M. Yamamoto, Appl. Phys. Lett. 95, 232512 (2009).

[10] M. Yamamoto, T. Ishikawa, T. Taira, G.-f. Li, K.-i. Matsuda, and T. Uemura, J. Phys.: Condens. Matter 22, 164212 (2010).

[11] H.-x. Liu, Y. Honda, T. Taira, K.-i. Matsuda, M. Arita, T. Uemura, and M. Yamamoto, Appl. Phys. Lett. 101, 132418 (2012).

[12] G.-f. Li, Y. Honda, H.-x. Liu, K.-i. Matsuda, M. Arita, T. Uemura, M. Yamamoto, Y. Miura, M. Shirai, T. Saito, F. Shi, and P. M. Voyles, Phys. Rev. B 89, 014428 (2014).

[13] N. Tezuka, N. Ikeda, F. Mitsuhashi, and S. Sugimoto, Appl. Phys. Lett. 94, 162504 (2009).

[14] T. Marukame, T. Ishikawa, T. Taira, K.-i. Matsuda, T. Uemura, and M. Yamamoto, Phys. Rev. B 81, 134432 (2010).

[15] W. Wang, E. Liu, M. Kodzuka, H. Sukegawa, M. Wojcik, E. Jedryka, G. H. Wu, K. Inomata, S. Mitani, and K. Hono, Phys. Rev. B 81, 140402(R) (2010).

[16] H.-x. Liu, T. Kawami, K. Moges, T. Uemura, M. Yamamoto, F. Shi, and P. M. Voyles, J. Phys. D: Appl. Phys. 48, 164001 (2015).

[17] K. Moges, Y. Honda, H.-x. Liu, T. Uemura, M. Yamamoto, Y. Miura, and M. Shirai, Phys. Rev. B 93, 134403 (2016).

[18] K. Yakushiji, K. Saito, S. Mitani, K. Takanashi, Y. K. Takahashi, and K. Hono, Appl. Phys. Lett. 88, 222504 (2006).

[19] T. Furubayashi, K. Kodama, H. Sukegawa, Y. K. Takahashi, K. Inomata, and K. Hono, Appl. Phys. Lett. 93, 122507 (2008).

[20] Y. Sakuraba, K. Izumi, T. Iwase, S. Bosu, K. Saito, K. Takanashi, Y. Miura, K. Futatsukawa, K. Abe, and M. Shirai, Phys. Rev. B 82, 094444 (2010).

[21] H. Narisawa, T. Kubota, and K. Takanashi, Appl. Phys. Express 8, 063008 (2015).

[22] Y. Du, T. Furubayashi, T. T. Sasaki, Y. Sakuraba, Y. K. Takahashi, and K. Hono, Appl. Phys. Lett. 107, 112405 (2015).

[23] T. Akiho, J. Shan, H.-x. Liu, K.-i. Matsuda, M. Yamamoto, and T. Uemura, Phys. Rev. B 87, 235205 (2013).

[24] P. Bruski, Y. Manzke, R. Farshchi, O. Brandt, J. Herfort, and M. Ramsteiner, Appl. Phys. Lett. 103, 052406 (2013).

[25] T. Saito, N. Tezuka, M. Matsuura, and S. Sugimoto, Appl. Phys. Express 6, 103006 (2013).

[26] Y. Ebina, T. Akiho, H.-x. Liu, M. Yamamoto, and T. Uemura, Appl. Phys. Lett. 104, 172405 (2014).
[27] T. Uemura, T. Akiho, Y. Ebina, and M. Yamamoto, Phys. Rev. B 91, 140410(R) (2015).

[28] J. Kübler, A. R. Williams, and C. B. Sommers, Phys. Rev. B 28, 1745 (1983).

[29] S. Ishida, S. Fujii, S. Kashiwagi, and S. Asano, J. Phys. Soc. Jpn. 64, 2152 (1995).

[30] S. Picozzi, A. Continenza, and A. J. Freeman, Phys. Rev. B 66, 094421 (2002).

[31] I. Galanakis, P. H. Dederichs, and N. Papanikolaou, Phys. Rev. B 66, 174429 (2002).

[32] P. J. Webster, J. Phys. Chem. Solids 32, 1221 (1971).

[33] S. Picozzi, A. Continenza, and A. J. Freeman, Phys. Rev. B 69, 094423 (2004).

[34] I. Galanakis, K. Özdoğan, B. Aktaş, and E. Şaşıŏlu, Appl. Phys. Lett. 89, 042502 (2006).

[35] B. Hülsen, M. Scheffler, and P. Kratzer, Phys. Rev. B 79, 094407 (2009).

[36] K. Miyamoto, A. Kimura, Y. Miura, M. Shirai, M. Ye, Y. Cui, K. Shimada, H. Namatame, M. Taniguchi, Y. Takeda, Y. Saitoh, E. Ikenaga, S. Ueda, K. Kobayashi, and T. Kanomata, Phys. Rev. B 79, 100405 (2009).

[37] T. Saito, T. Katayama, T. Ishikawa, M. Yamamoto, D. Asakura, T. Koide, Y. Miura, and M. Shirai, Phys. Rev. B 81, 144417 (2010).

[38] Y. Miura, K. Abe, and M. Shirai, Phys. Rev. B 83, 214411 (2011)

[39] R. Fetzer, J.-P. Wüstenberg, T. Taira, T. Uemura, M. Yamamoto, M. Aeschlimann, and M. Cinchetti, Phys. Rev. B 87, 184418 (2013).

[40] M. Jourdan, J. Minár, J. Braun, A. Kronenberg, S. Chadov, B. Balke, A. Gloskovskii, M. Kolbe, H. J. Elmers, G. Schönhense, H. Ebert, C. Felser, and M. Kläui, Nat. Commun. 5, 3974 (2014).

[41] R. Fetzer, B. Stadtmüller, Y. Ohdaira, H. Naganuma, M. Oogane, Y. Ando, T. Taira, T. Uemura, M. Yamamoto, M. Aeschlimann, and M. Cinchetti, Sci. Rep. 5, 8537 (2015).

[42] J.-P. Wüstenberg, R. Fetzer, M. Aeschlimann, M. Cinchetti, J. Minár, Jürgen Braun, H. Ebert, T. Ishikawa, T. Uemura, and M. Yamamoto, Phys. Rev. B 85, 064407 (2012).

[43] D. Asakura, T. Koide, S. Yamamoto, K. Tsuchiya, T. Shioya, K. Amemiya, V. R. Singh, T. Kataoka, Y. Yamazaki, Y. Sakamoto, A. Fujimori, T. Taira, and M. Yamamoto, Phys. Rev. B 82, 184419 (2010).

[44] V. R. Singh, V. K. Verma, K. Ishigami, G. Shibata, T. Kadono, A. Fujimori, D. Asakura, T. Koide, Y. Miura, M. Shirai, G.-f. Li, T. Taira, and M. Yamamoto, Phys. Rev. B 86, 144412 (2012).

[45] V. R. Singh, V. K. Verma, K. Ishigami, G. Shibata, A. Fujimori, T. Koide, Y. Miura, M. Shirai, T. Ishikawa, G. -f. Li, and M. Yamamoto, J. Appl. Phys. 117, 203901 (2015).

[46] S. Ouardi, G. H. Fecher, S. Chadov, B. Balke, X. Kozina, C. Felser, T. Taira, and M. Yamamoto, Appl. Phys. A: Mater. Sci. Process. 111, 395 (2013).

[47] X. Kozina, J. Karel, S. Ouardi, S. Chadov, G. H. Fecher, C. Felser, G. Stryganyuk, B. Balke, T. Ishikawa, T. Uemura, M. Yamamoto, E. Ikenaga, S. Ueda, and K. Kobayashi, Phys. Rev. B 89, 125116 (2014).

[48] R. Fetzer, S. Ouardi, Y. Honda, H.-x. Liu, S. Chadov, B. Balke, S. Ueda, M. Suzuki, T. Uemura, M. Yamamoto, M. Aeschlimann, M. Cinchetti, G. H. Fecher, and C. Felser, J. Phys. D: Appl. Phys. 48, 164002 (2015).

[49] S. Zhang, P. M. Levy, A. C. Marley, and S. S. P. Parkin, Phys. Rev. Lett. 79, 3744 (1997). 
[50] C. H. Shang, J. Nowak, R. Jansen, and J. S. Moodera, Phys. Rev. B 58, R2917 (1998).

[51] X.-F. Han, A. C. C. Yu, M. Oogane, J. Murai, T. Daibou, and T. Miyazaki, Phys. Rev. B 63, 224404 (2001).

[52] V. Drewello, J. Schmalhorst, A. Thomas, and G. Reiss, Phys. Rev. B 77, 014440 (2008).

[53] R. Shan, H. Sukegawa, W. H. Wang, M. Kodzuka, T. Furubayashi, T. Ohkubo, S. Mitani, K. Inomata, and K. Hono, Phys. Rev. Lett. 102, 246601 (2009).

[54] S. S. P. Parkin, C. Kaiser, A. Panchula, P. M. Rice, B. Hughes, M. Samant, and S.-H. Yang, Nat. Mater. 3, 862 (2004).

[55] T. Marukame and M. Yamamoto, J. Appl. Phys. 101, 083906 (2007).

[56] A. A. Khan, J. Schmalhorst, G. Reiss, G. Eilers, M. Münzenberg, H. Schuhmann, and M. Seibt, Phys. Rev. B 82, 064416 (2010).

[57] V. Drewello, D. Ebke, M. Schäfers, Z. Kugler, G. Reiss, and A. Thomas, J. Appl. Phys. 111, 07 C701 (2012).

[58] D. Bang, T. Nozaki, D. D. Djayaprawira, M. Shiraishi, Y. Suzuki, A. Fukushima, H. Kubota, T. Nagahama, S. Yuasa, H. Maehara,
K. Tsunekawa, Y. Nagamine, N. Watanabe, and H. Itoh, J. Appl. Phys. 105, 07C924 (2009).

[59] Q. L. Ma, S. G. Wang, J. Zhang, Y. Wang, R. C. C. Ward, C. Wang, A. Kohn, X.-G. Zhang, and X. F. Han, Appl. Phys. Lett. 95, 052506 (2009).

[60] M. Belmoubarik, H. Sukegawa, T. Ohkubo, S. Mitani, and K. Hono, Appl. Phys. Lett. 108, 132404 (2016).

[61] R. Stratton, J. Phys. Chem. Solids 23, 1177 (1962).

[62] T. Marukame, T. Ishikawa, W. Sekine, K. Matsuda, T. Uemura, and M. Yamamoto, IEEE Trans. Magn. 42, 2652 (2006).

[63] S. Yuasa, T. Nagahama, A. Fukushima, Y. Suzuki, and K. Ando, Nat. Mater. 3, 868 (2004).

[64] M. Julliere, Phys. Lett. A 54, 225 (1975).

[65] W. H. Butler, X.-G. Zhang, T. C. Schulthess, and J. M. MacLaren, Phys. Rev. B 63, 054416 (2001).

[66] J. Mathon and A. Umerski, Phys. Rev. B 63, 220403(R) (2001).

[67] Y. Miura, H. Uchida, Y. Oba, K. Nagao, and M. Shirai, J. Phys.: Condens. Matter 19, 365228 (2007).

[68] S. Trudel, O. Gaier, J. Hamrle, and B. Hillebrands, J. Phys. D: Appl. Phys. 43, 193001 (2010). 